COMMUNICATIONS IN

ANALYSIS AND GEOMETRY

Volume 11, Number 5, 859-907, 2003

\title{
Singularities of special Lagrangian fibrations and the SYZ Conjecture
}

\author{
DOMinic JoYCe
}

\begin{abstract}
The SYZ Conjecture explains Mirror Symmetry between mirror Calabi-Yau 3-folds $M, \hat{M}$ in terms of special Lagrangian fibrations $f: M \rightarrow B$ and $\hat{f}: \hat{M} \rightarrow B$ over the same base $B$, whose fibres are dual 3-tori, except for singular fibres. This paper studies the singularities of special Lagrangian fibrations.

We construct many examples of special Lagrangian fibrations on open subsets of $\mathbb{C}^{3}$. The simplest are given explicitly, and the rest use analytic existence results for $\mathrm{U}(1)$-invariant special Lagrangian 3 -folds in $\mathbb{C}^{3}$. We then argue that some features of our examples should also hold for generic special Lagrangian fibrations of (almost) Calabi-Yau 3-folds, and draw some conclusions on the SYZ Conjecture.
\end{abstract}

\section{Introduction.}

In 1996, Strominger, Yau and Zaslow [23] suggested a geometrical interpretation of Mirror Symmetry between Calabi-Yau 3-folds $M, \hat{M}$ in terms of dual fibrations by special Lagrangian 3-tori, now known as the $S Y Z$ Conjecture. Here is an attempt to state it.

The SYZ Conjecture. Suppose $M$ and $\hat{M}$ are mirror Calabi-Yau 3folds. Then (under some additional conditions) there should exist a compact topological 3-manifold $B$ and surjective, continuous maps $f: M \rightarrow B$ and $\hat{f}: \hat{M} \rightarrow B$, such that

(i) There exists a dense open set $B_{0} \subset B$, such that for each $b \in B_{0}$, the fibres $f^{-1}(b)$ and $\hat{f}^{-1}(b)$ are nonsingular special Lagrangian 3 -tori $T^{3}$ in $M$ and $\hat{M}$. Furthermore, $f^{-1}(b)$ and $\hat{f}^{-1}(b)$ are in some sense dual to one another.

(ii) For each $b \in \Delta=B \backslash B_{0}$, the fibres $f^{-1}(b)$ and $\hat{f}^{-1}(b)$ are expected to be singular special Lagrangian 3 -folds in $M$ and $\hat{M}$. 
We call $f$ and $\hat{f}$ special Lagrangian fibrations, and $f^{-1}(b), \hat{f}^{-1}(b)$ for $b \in \Delta$ the singular fibres. In this paper we consider the question: what is the nature of the 'singular fibres' of the fibration, and what do $f, \hat{f}$ look like near the singularities?

The main rigorous results of the paper are the construction and study in $\S 5-\S 7$ of examples of special Lagrangian fibrations on open subsets of $\mathbb{C}^{3}$. The fibrations of $\S 5$ are completely explicit, but those in $\S 6$ and $\S 7$ are constructed using analytic existence results from the author's series of papers $[9,10,11]$ on special Lagrangian 3 -folds in $\mathbb{C}^{3}$ invariant under the $\mathrm{U}(1)$-action

$$
\mathrm{e}^{i \theta}:\left(z_{1}, z_{2}, z_{3}\right) \longmapsto\left(\mathrm{e}^{i \theta} z_{1}, \mathrm{e}^{-i \theta} z_{2}, z_{3}\right) \text { for } \mathrm{e}^{i \theta} \in \mathrm{U}(1) .
$$

However, the heart of the paper is not the rigorous results but the discussion and conjecture in $\S 5.1, \S 6.1, \S 7.4$ and $\S 8$. Here we argue, with justifications but not full proofs, that various features of our examples should also be true of special Lagrangian fibrations of (almost) Calabi-Yau 3-folds, especially in the generic case.

In particular, we claim that special Lagrangian fibrations $f: M \rightarrow B$ will in general not be smooth but only piecewise smooth, and that the discriminant $\Delta$ of $f$ is of real codimension one in $B$ and is typically made up of 'ribbons'. We use this to argue that the version of the SYZ Conjecture above is too strong, because the discriminants $\Delta, \hat{\Delta}$ of $f, \hat{f}$ are not homeomorphic, and so cannot coincide in $B$.

The paper was originally motivated by the work of Gross $[3,4,5]$ and Ruan [20, 21, 22]. The first version, the preprint math.DG/0011179 in November 2000, consisted mainly of conjectures, and so was not suitable for publication. It was concerned to refute the widespread assumption in early papers on the SYZ Conjecture that special Lagrangian fibrations would be smooth.

In this second version I have used the results of $[9,10,11]$ to prove many of the conjectures in the first version. I have also reduced the emphasis on smoothness of fibrations, as I feel the field has moved on from two years ago and there is no longer a need to argue the case.

We begin in $\S 2$ and $\S 3$ by introducing special Lagrangian geometry and special Lagrangian fibrations. Section 4 reviews the main results of $[9,10,11]$ on U(1)-invariant special Lagrangian 3 -folds in $\mathbb{C}^{3}$. The new material is $\S 5$ $\S 8$. Section 5 defines two explicit special Lagrangian fibrations $F, F^{\prime}: \mathbb{C}^{3} \rightarrow$ $\mathbb{R}^{3}$ with singular fibres in codimension 1 in $\mathbb{R}^{3}$.

Section 6 defines a more complicated special Lagrangian fibration $\hat{F}$ : $V \rightarrow \mathbb{R}^{3}$ which models a certain kind of singular behaviour in codimension 
2 in $\mathbb{R}^{3}$. Section 7 constructs a continuous 1 -parameter family of special Lagrangian fibrations $F^{t}: V \rightarrow \mathbb{R}^{3}$ for $t \in[0,1]$, where $F^{0}$ is smooth, but $F^{t}$ is not smooth for $t \in(0,1]$. Thus the $F^{t}$ model how to deform smooth special Lagrangian fibrations to non-smooth ones.

In each of $\S 5-\S 7$ we also discuss what features of our examples we expect to hold for special Lagrangian fibrations of (almost) Calabi-Yau 3-folds, and why. Finally, in $\S 8$ we explain the picture of smooth special Lagrangian fibrations built up by Gross and Ruan, and by considering the changes as we deform from a smooth to a generic fibration, we draw some conclusions on the SYZ Conjecture.

Acknowledgements. I would like to thank Mark Gross, Richard Thomas, Nigel Hitchin and David Morrison for helpful conversations. I was supported by an EPSRC Advanced Research Fellowship whilst writing this paper.

\section{Special Lagrangian geometry.}

We now introduce the idea of special Lagrangian submanifolds (SL $m$-folds), in two different geometric contexts. First, in $\S 2.1$, we define SL $m$-folds in $\mathbb{C}^{m}$. Then $\S 2.2$ discusses SL $m$-folds in almost Calabi-Yau $m$-folds, compact Kähler manifolds equipped with a holomorphic volume form which generalize the idea of Calabi-Yau manifolds. Finally, section 2.3 considers the singularities of SL $m$-folds. The principal references for this section are Harvey and Lawson [7] and the author [8, 17].

\subsection{Special Lagrangian submanifolds in $\mathbb{C}^{m}$.}

We begin by defining calibrations and calibrated submanifolds, following Harvey and Lawson [7].

Definition 2.1. Let $(M, g)$ be a Riemannian manifold. An oriented tangent $k$-plane $V$ on $M$ is a vector subspace $V$ of some tangent space $T_{x} M$ to $M$ with $\operatorname{dim} V=k$, equipped with an orientation. If $V$ is an oriented tangent $k$-plane on $M$ then $\left.g\right|_{V}$ is a Euclidean metric on $V$, so combining $\left.g\right|_{V}$ with the orientation on $V$ gives a natural volume form $\operatorname{vol}_{V}$ on $V$, which is a $k$-form on $V$.

Now let $\varphi$ be a closed $k$-form on $M$. We say that $\varphi$ is a calibration on $M$ if for every oriented $k$-plane $V$ on $M$ we have $\left.\varphi\right|_{V} \leqslant \operatorname{vol}_{V}$. Here $\left.\varphi\right|_{V}=\alpha \cdot \operatorname{vol}_{V}$ for some $\alpha \in \mathbb{R}$, and $\left.\varphi\right|_{V} \leqslant \operatorname{vol}_{V}$ if $\alpha \leqslant 1$. Let $N$ be an oriented submanifold of $M$ with dimension $k$. Then each tangent space $T_{x} N$ 
for $x \in N$ is an oriented tangent $k$-plane. We say that $N$ is a calibrated submanifold if $\left.\varphi\right|_{T_{x} N}=\operatorname{vol}_{T_{x} N}$ for all $x \in N$.

It is easy to show that calibrated submanifolds are automatically minimal submanifolds [7, Th. II.4.2]. Here is the definition of special Lagrangian submanifolds in $\mathbb{C}^{m}$, taken from $[7, \S \mathrm{III}]$.

Definition 2.2. Let $\mathbb{C}^{m}$ have complex coordinates $\left(z_{1}, \ldots, z_{m}\right)$, and define a metric $g^{\prime}$, a real 2 -form $\omega^{\prime}$ and a complex $m$-form $\Omega^{\prime}$ on $\mathbb{C}^{m}$ by

$$
\begin{aligned}
g^{\prime}=\left|\mathrm{d} z_{1}\right|^{2}+\cdots+\left|\mathrm{d} z_{m}\right|^{2}, & \omega^{\prime}=\frac{i}{2}\left(\mathrm{~d} z_{1} \wedge \mathrm{d} \bar{z}_{1}+\cdots+\mathrm{d} z_{m} \wedge \mathrm{d} \bar{z}_{m}\right), \\
\text { and } \quad & \Omega^{\prime}=\mathrm{d} z_{1} \wedge \cdots \wedge \mathrm{d} z_{m} .
\end{aligned}
$$

Then $\operatorname{Re} \Omega^{\prime}$ and $\operatorname{Im} \Omega^{\prime}$ are real $m$-forms on $\mathbb{C}^{m}$. Let $L$ be an oriented real submanifold of $\mathbb{C}^{m}$ of real dimension $m$. We say that $L$ is a special Lagrangian submanifold of $\mathbb{C}^{m}$, or $S L m$-fold for short, if $L$ is calibrated with respect to $\operatorname{Re} \Omega^{\prime}$, in the sense of Definition 2.1.

Harvey and Lawson [7, Cor. III.1.11] give the following alternative characterization of special Lagrangian submanifolds:

Proposition 2.3. Let $L$ be a real m-dimensional submanifold of $\mathbb{C}^{m}$. Then $L$ admits an orientation making it into an SL submanifold of $\mathbb{C}^{m}$ if and only if $\left.\omega^{\prime}\right|_{L} \equiv 0$ and $\left.\operatorname{Im} \Omega^{\prime}\right|_{L} \equiv 0$.

An $m$-dimensional submanifold $L$ in $\mathbb{C}^{m}$ is called Lagrangian if $\left.\omega^{\prime}\right|_{L} \equiv 0$. Thus special Lagrangian submanifolds are Lagrangian submanifolds satisfying the extra condition that $\left.\operatorname{Im} \Omega^{\prime}\right|_{L} \equiv 0$, which is how they get their name.

\subsection{Almost Calabi-Yau $m$-folds and SL $m$-folds.}

We shall define special Lagrangian submanifolds not just in Calabi-Yau manifolds, as usual, but in the much larger class of almost Calabi-Yau manifolds.

Definition 2.4. Let $m \geqslant 2$. An almost Calabi-Yau $m$-fold, or $A C Y m$ fold for short, is a quadruple $(M, J, \omega, \Omega)$ such that $(M, J)$ is a compact $m$-dimensional complex manifold, $\omega$ is the Kähler form of a Kähler metric $g$ on $M$, and $\Omega$ is a non-vanishing holomorphic $(m, 0)$-form on $M$.

We call $(M, J, \omega, \Omega)$ a Calabi-Yau m-fold, or $C Y m$-fold for short, if in addition $\omega$ and $\Omega$ satisfy

$$
\omega^{m} / m !=(-1)^{m(m-1) / 2}(i / 2)^{m} \Omega \wedge \bar{\Omega} .
$$


Then for each $x \in M$ there exists an isomorphism $T_{x} M \cong \mathbb{C}^{m}$ that identifies $g_{x}, \omega_{x}$ and $\Omega_{x}$ with the flat versions $g^{\prime}, \omega^{\prime}, \Omega^{\prime}$ on $\mathbb{C}^{m}$ in (1). Furthermore, $g$ is Ricci-flat and its holonomy group is a subgroup of $\mathrm{SU}(m)$.

This is not the usual definition of a Calabi-Yau manifold, but is essentially equivalent to it.

Definition 2.5. Let $(M, J, \omega, \Omega)$ be an almost Calabi-Yau $m$-fold, and $N$ a real $m$-dimensional submanifold of $M$. We call $N$ a special Lagrangian submanifold, or $S L m$-fold for short, if $\left.\left.\omega\right|_{N} \equiv \operatorname{Im} \Omega\right|_{N} \equiv 0$. It easily follows that $\left.\operatorname{Re} \Omega\right|_{N}$ is a nonvanishing $m$-form on $N$. Thus $N$ is orientable, with a unique orientation in which $\left.\operatorname{Re} \Omega\right|_{N}$ is positive.

Again, this is not the usual definition of special Lagrangian submanifold, but is essentially equivalent to it. When $(M, J, \omega, \Omega)$ is a Calabi-Yau $m$ fold, $N$ is special Lagrangian if and only if it is calibrated w.r.t. Re $\Omega$. More generally $[8, \S 9.5]$, SL $m$-folds in an ACY $m$-fold are calibrated w.r.t. Re $\Omega$, but for a suitably conformally rescaled metric $g$.

Thus, we could define SL $m$-folds in ACY $m$-folds using calibrated geometry, as in Definition 2.2. But in the author's view the definition of SL $m$-folds using the vanishing of closed forms is more fundamental than that using calibrated geometry, and so should be taken as the primary definition.

The deformation theory of special Lagrangian submanifolds was studied by McLean $[18, \S 3]$, who proved the following result in the Calabi-Yau case. The extension to the ACY case is described in $[8, \S 9.5]$.

Theorem 2.6. Let $(M, J, \omega, \Omega)$ be an almost Calabi-Yau m-fold, and $N$ a compact $S L m$-fold in $M$. Then the moduli space $\mathcal{M}_{N}$ of special Lagrangian deformations of $N$ is a smooth manifold of dimension $b^{1}(N)$, the first Betti number of $N$.

Using similar methods one can prove $[8, \S 9.3, \S 9.5]$ :

Theorem 2.7. Let $\left\{\left(M, J_{t}, \omega_{t}, \Omega_{t}\right): t \in(-\epsilon, \epsilon)\right\}$ be a smooth 1-parameter family of almost Calabi-Yau m-folds. Suppose $N_{0}$ is a compact SL m-fold in $\left(M, J_{0}, \omega_{0}, \Omega_{0}\right)$, with $\left[\left.\omega_{t}\right|_{N_{0}}\right]=0$ in $H^{2}\left(N_{0}, \mathbb{R}\right)$ and $\left[\left.\operatorname{Im} \Omega_{t}\right|_{N_{0}}\right]=0$ in $H^{m}\left(N_{0}, \mathbb{R}\right)$ for all $t \in(-\epsilon, \epsilon)$. Then $N_{0}$ extends to a smooth 1-parameter family $\left\{N_{t}: t \in(-\delta, \delta)\right\}$, where $0<\delta \leqslant \epsilon$ and $N_{t}$ is a compact SL m-fold in $\left(M, J_{t}, \omega_{t}, \Omega_{t}\right)$. 


\subsection{Singularities of SL $m$-folds.}

We now summarize some results on singularities of SL $m$-folds, taken from the survey [17] of a series of papers $[13,14,15,16]$ by the author. We start with a definition on $S L$ cones in $\mathbb{C}^{m}$, adapted from [17, §3.1].

Definition 2.8. A (singular) SL $m$-fold $C$ in $\mathbb{C}^{m}$ is called a cone if $C=t C$ for all $t>0$, where $t C=\{t \mathbf{x}: \mathbf{x} \in C\}$. Let $C$ be a closed SL cone in $\mathbb{C}^{m}$ for $m>2$ with an isolated singularity at 0 . Then $\Sigma=C \cap \mathcal{S}^{2 m-1}$ is a compact, nonsingular $(m-1)$-submanifold of $\mathcal{S}^{2 m-1}$, not necessarily connected. Let $g_{\Sigma}$ be the restriction of $g^{\prime}$ to $\Sigma$, where $g^{\prime}$ is as in (1).

Let $\Delta_{\Sigma}$ be the Laplacian on $\left(\Sigma, g_{\Sigma}\right)$. Define

$$
\mathcal{D}_{\Sigma}=\left\{\alpha \in \mathbb{R}: \alpha(\alpha+m-2) \text { is an eigenvalue of } \Delta_{\Sigma}\right\} .
$$

Then $\mathcal{D}_{\Sigma}$ is a countable, discrete subset of $\mathbb{R}$. Let $N(\Sigma)$ be the number of eigenvalues of $\Delta_{\Sigma}$ in $(0,2 m]$, counted with multiplicity. Let $G$ be the Lie subgroup of $\mathrm{SU}(m)$ preserving $C$. Define the stability index s-ind $(C)$ to be

$$
\operatorname{s-ind}(C)=N(\Sigma)-m^{2}-2 m+1+\operatorname{dim} G .
$$

Then s-ind $(C) \geqslant 0$. We call $C$ stable if s-ind $(C)=0$.

In $[17$, Def. 3.7] we define conical singularities of SL $m$-folds.

Definition 2.9. Let $(M, J, \omega, \Omega)$ be an almost Calabi-Yau $m$-fold for $m>$ 2. Suppose $X$ is a compact singular SL $m$-fold in $M$ with singularities at distinct points $x_{1}, \ldots, x_{n} \in X$, and no other singularities. Fix isomorphisms $v_{i}: \mathbb{C}^{m} \rightarrow T_{x_{i}} M$ for $i=1, \ldots, n$ such that $v_{i}^{*}(\omega)=\omega^{\prime}$ and $v_{i}^{*}(\Omega)=c_{i} \Omega^{\prime}$, where $\omega^{\prime}, \Omega^{\prime}$ are as in (1) and $c_{i}>0$.

Let $C_{1}, \ldots, C_{n}$ be SL cones in $\mathbb{C}^{m}$ with isolated singularities at 0 . For $i=1, \ldots, n$ let $\Sigma_{i}=C_{i} \cap \mathcal{S}^{2 m-1}$, and let $\mu_{i} \in(2,3)$ with $\left(2, \mu_{i}\right] \cap \mathcal{D}_{\Sigma_{i}}=\emptyset$, where $\mathcal{D}_{\Sigma_{i}}$ is defined in (3). Then we say that $X$ has a conical singularity at $x_{i}$, with rate $\mu_{i}$ and cone $C_{i}$ for $i=1, \ldots, n$, if the following holds.

By Darboux' Theorem there exist embeddings $\Upsilon_{i}: B_{R} \rightarrow M$ for $i=$ $1, \ldots, n$ satisfying $\Upsilon_{i}(0)=x_{i},\left.\mathrm{~d} \Upsilon_{i}\right|_{0}=v_{i}$ and $\Upsilon_{i}^{*}(\omega)=\omega^{\prime}$, where $B_{R}$ is the open ball of radius $R$ about 0 in $\mathbb{C}^{m}$ for some small $R>0$. Define $\iota_{i}: \Sigma_{i} \times(0, R) \rightarrow B_{R}$ by $\iota_{i}(\sigma, r)=r \sigma$ for $i=1, \ldots, n$.

Define $X^{\prime}=X \backslash\left\{x_{1}, \ldots, x_{n}\right\}$. Then there should exist a compact subset $K \subset X^{\prime}$ such that $X^{\prime} \backslash K$ is a union of open sets $S_{1}, \ldots, S_{n}$ with $S_{i} \subset \Upsilon_{i}\left(B_{R}\right)$, whose closures $\bar{S}_{1}, \ldots, \bar{S}_{n}$ are disjoint in $X$. For $i=1, \ldots, n$ and some 
$R^{\prime} \in(0, R]$ there should exist a smooth $\phi_{i}: \Sigma_{i} \times\left(0, R^{\prime}\right) \rightarrow B_{R}$ such that $\Upsilon_{i} \circ \phi_{i}: \Sigma_{i} \times\left(0, R^{\prime}\right) \rightarrow M$ is a diffeomorphism $\Sigma_{i} \times\left(0, R^{\prime}\right) \rightarrow S_{i}$, and

$$
\left|\nabla^{k}\left(\phi_{i}-\iota_{i}\right)\right|=O\left(r^{\mu_{i}-1-k}\right) \quad \text { as } r \rightarrow 0 \text { for } k=0,1 \text {. }
$$

Here $\nabla$ is the Levi-Civita connection of the cone metric $\iota_{i}^{*}\left(g^{\prime}\right)$ on $\Sigma_{i} \times\left(0, R^{\prime}\right)$, $|$.$| is computed using \iota_{i}^{*}\left(g^{\prime}\right)$. If the cones $C_{1}, \ldots, C_{n}$ are stable in the sense of Definition 2.8, then we say that $X$ has stable conical singularities.

In [14] we study moduli spaces of SL $m$-folds with conical singularities. The case when $C_{i}$ are stable is particularly simple, [14, Cor. 6.11]:

Theorem 2.10. Suppose $(M, J, \omega, \Omega)$ is an almost Calabi-Yau m-fold and $X$ a compact $S L m$-fold in $M$ with stable conical singularities $x_{1}, \ldots, x_{n}$. Let $\mathcal{M}_{X}$ be the moduli space of deformations of $X$ as an $S L$ m-fold with conical singularities in $M$. Set $X^{\prime}=X \backslash\left\{x_{1}, \ldots, x_{n}\right\}$, and let $\mathcal{I}_{X^{\prime}}$ be the image of $H_{\mathrm{cs}}^{1}\left(X^{\prime}, \mathbb{R}\right)$ in $H^{1}\left(X^{\prime}, \mathbb{R}\right)$. Then $\mathcal{M}_{X}$ is a smooth manifold of $d i$ mension $\operatorname{dim} \mathcal{I}_{X^{\prime}}$.

Here $H_{\mathrm{cs}}^{k}\left(X^{\prime}, \mathbb{R}\right), H^{k}\left(X^{\prime}, \mathbb{R}\right)$ are the compactly-supported and usual de Rham cohomology groups of $X^{\prime}$. Note the similarity with Theorem 2.6. In [14, Cor. 7.10], Theorem 2.10 is extended to moduli spaces in families of almost Calabi-Yau $m$-folds $\left(M, J^{s}, \omega^{s}, \Omega^{s}\right)$. It implies that SL $m$-folds $X$ with stable conical singularities persist under small deformations of $(M, J, \omega, \Omega)$ satisfying some necessary cohomological conditions.

In $[15,16]$ we study desingularizations of SL $m$-folds with conical singularities. Here is the basic idea. Let $M, X$ and $x_{i}, C_{i}$ for $i=1, \ldots, n$ be as in Definition 2.9. Let $L_{i}$ be an Asymptotically Conical $S L m$-fold in $\mathbb{C}^{m}$, asymptotic to $C_{i}$ at infinity. Then $t L_{i}=\left\{t \mathbf{x}: \mathbf{x} \in L_{i}\right\}$ is also asymptotic to $C_{i}$ for all $t>0$.

We explicitly construct a 1-parameter family of compact, nonsingular Lagrangian $m$-folds $N^{t}$ in $(M, \omega)$ for $t \in(0, \delta)$ by gluing $t L_{i}$ into $X$ at $x_{i}$, using a partition of unity. Then we prove using analysis that for small $t \in(0, \delta)$ we can deform $N^{t}$ to a special Lagrangian $m$-fold $\tilde{N}^{t}$ in $M$, so that $\tilde{N}^{t} \rightarrow X$ as $t \rightarrow 0$ in the sense of currents.

The results are complicated, so we will not reproduce them. Interested readers are advised to consult $[17, \S 7]$. From them we deduce results on desingularizing SL 3 -folds with $T^{2}$-cone singularities in $[17, \S 10]$, which will provide partial proofs of Conjectures 5.5 and 6.7. 


\section{Introduction to special Lagrangian fibrations.}

We begin by defining special Lagrangian fibrations, following [4, Def. 1.4].

Definition 3.1. Let $(M, J, \omega, \Omega)$ be an almost Calabi-Yau $m$-fold, and $B$ a Hausdorff topological space. We call $f: M \rightarrow B$ a special Lagrangian fibration if $f$ is a continuous, surjective map, and for all $b \in B, f^{-1}(b)$ is the support of a special Lagrangian integral current $T$ in $M$ with $\partial T=0$.

Here integral currents are meant in the sense of Geometric Measure Theory. They are a measure-theoretic generalization of submanifold, including singular submanifolds. Harvey and Lawson [7, $\S \mathrm{I}]$ frame their discussion of calibrated geometry in terms of currents, and define calibrated integral currents as well as calibrated submanifolds. For an introduction to Geometric Measure Theory, see Morgan [19].

We shall not use much Geometric Measure Theory in this paper. The point to note is that the fibres $f^{-1}(b)$ are compact SL $m$-folds in $M$ without boundary, which may have singularities of a fairly general kind.

Definition 3.2. Let $(M, J, \omega, \Omega)$ be an almost Calabi-Yau $m$-fold, and $f$ : $M \rightarrow B$ a special Lagrangian fibration. The fibres $N_{b}$ of $f$ are $N_{b}=f^{-1}(b)$ for $b \in B$. We call a fibre $N_{b}$ nonsingular if $B$ has the structure of a smooth real $m$-manifold near $b$, and $f: M \rightarrow B$ is a smooth submersion along $N_{b}$. That is, for each $x \in N_{b}$ the map $\mathrm{d}_{x} f: T_{x} M \rightarrow T_{b} B$ is surjective. Otherwise we call $N_{b}$ a singular fibre. Define the discriminant of $f$ to be $\Delta=\left\{b \in B: N_{b}\right.$ is a singular fibre $\}$. Roughly speaking, $\Delta$ is the set of singular fibres, and $B \backslash \Delta$ the set of nonsingular fibres. It is easy to show that $B \backslash \Delta$ is open in $B$, and so $\Delta$ is closed in $B$.

Note that singular fibres $N_{b}$ may actually sometimes be nonsingular submanifolds of $M$. For instance, one can write down an explicit SL fibration of $T^{6} / \mathbb{Z}_{2}$ including 1-parameter families of singular fibres $T^{3} / \mathbb{Z}_{2}$, which are nonsingular as 3-submanifolds, but are 'double fibres' of the fibration.

Using action angle coordinates, Duistermaat [1, Th. 1.1] proves:

Proposition 3.3. Let $(M, J, \omega, \Omega)$ be an almost Calabi-Yau m-fold, and $f: M \rightarrow B$ a special Lagrangian fibration. Then each connected component of a nonsingular fibre $N_{b}$ is a nonsingular submanifold of $M$ diffeomorphic to $T^{m}$. 
The basic idea is that on a nonsingular fibre $N_{b}$ one can define a natural action of $T_{b}^{*} B=\mathbb{R}^{m}$, which turns out to be transitive on connected components. Now arbitrary SL fibrations $f: M \rightarrow B$ are difficult to study, as we have little control over their singular behaviour. So it is helpful to add extra simplifying assumptions. Two such assumptions we will consider in this paper are that $f$ is smooth, and that $f$ is generic.

Definition 3.4. Let $(M, J, \omega, \Omega)$ be an almost Calabi-Yau $m$-fold, and $f$ : $M \rightarrow B$ a special Lagrangian fibration. We call $f$ smooth if $B$ is a smooth real $m$-manifold, and $f$ a smooth map.

Smoothness has strong consequences for the structure of the discriminant $\Delta$. From Gross $[4, \S 1]$ we deduce:

Proposition 3.5. Let $M$ be an almost Calabi-Yau m-fold, and $f: M \rightarrow B$ a smooth special Lagrangian fibration. Then the discriminant $\Delta$ has Hausdorff codimension at least two in $B$.

His proof uses the fact that the fibres are both Lagrangian and minimal. The Lagrangian assumption is used to prove [4, Prop. 2.2] that if $x \in N_{b}$ and $\operatorname{rank}\left(\mathrm{d}_{x} f\right): T_{x} M \rightarrow T_{b} B$ is $k$, then $N_{b}$ contains a $k$-dimensional submanifold through $x$ on which $\operatorname{rank}(\mathrm{d} f)$ is $k$. But by a result of Almgren, the singularities of a minimal submanifold are of Hausdorff codimension at least two. Combining these two shows that $\operatorname{rank}\left(\mathrm{d}_{x} f\right)$ cannot be $m-1$, so that if $x$ is a singular point of $N_{b}$ then $\operatorname{rank}\left(\mathrm{d}_{x} f\right) \leqslant m-2$.

Using these ideas, one can show that if $f: M \rightarrow B$ is a smooth SL fibration of an almost Calabi-Yau 3-fold with discriminant $\Delta$, then under good circumstances we expect the following properties:

(i) $\Delta$ is a union $\Delta_{0} \cup \Delta_{1}$, where $\Delta_{0}$ is a finite set of points, and $\Delta_{1}$ a finite set of open intervals. Essentially, $\Delta$ is a graph in $B$.

(ii) For each $b \in \Delta_{1}$, the singular set of $N_{b}$ is a finite number of circles $\mathcal{S}^{1}$, and the singularities are locally modelled on $L \times \mathbb{R}$ in $\mathbb{C}^{2} \times \mathbb{C}$, where $L$ is a special Lagrangian 2-fold in $\mathbb{C}^{2}$ with an isolated singularity at 0 .

That is, singular fibres occur in codimension two in the base, and the generic singular fibre has a one-dimensional singular set. Next we define generic SL fibrations.

Definition 3.6. Let $(M, J, \omega, \Omega)$ be a Calabi-Yau or almost Calabi-Yau $m$ fold, and $f: M \rightarrow B$ a special Lagrangian fibration of $(M, J, \omega, \Omega)$. We shall 
say that some property of $f$ is generic if for all Kähler forms $\tilde{\omega}$ on $M$ in the same Kähler class as $\omega$ and sufficiently close to $\omega$, there exists close to $f$ a special Lagrangian fibration $\tilde{f}: M \rightarrow B$ of the almost Calabi-Yau $m$-fold $(M, J, \tilde{\omega}, \Omega)$ with the same property. Examples of properties of $f$ that might or might not be generic are: existence, smoothness, every singular fibre has only finitely many singular points, and so on.

Here is the reasoning behind this definition. We intend to call a property of a special Lagrangian fibration generic if it holds for fibrations of all nearby almost Calabi-Yau $m$-folds $(M, \tilde{J}, \tilde{\omega}, \tilde{\Omega})$. Now if $N$ is a nonsingular fibre of $f$, then Theorem 2.7 shows that the only obstructions to finding an SL $m$-fold in $(M, \tilde{J}, \tilde{\omega}, \tilde{\Omega})$ near $N$ are that $\left[\left.\tilde{\omega}\right|_{N}\right] \equiv\left[\left.\operatorname{Im} \tilde{\Omega}\right|_{N}\right] \equiv 0$.

To ensure this holds, we restrict attention to ACY $m$-folds $(M, \tilde{J}, \tilde{\omega}, \tilde{\Omega})$ with $[\tilde{\omega}]=[\omega]$ in $H^{2}(M, \mathbb{R})$ and $[\operatorname{Im} \tilde{\Omega}]=[\operatorname{Im} \Omega]$ in $H^{3}(M, \mathbb{R})$. But one can show that if $[\operatorname{Im} \tilde{\Omega}]=[\operatorname{Im} \Omega]$ and $(M, J, \Omega),(M, \tilde{J}, \tilde{\Omega})$ are close, then they are isomorphic. So we may as well fix $\tilde{J}=J$ and $\tilde{\Omega}=\Omega$, and just vary the Kähler form $\tilde{\omega}$ within the Kähler class of $\omega$.

\section{U(1)-invariant special Lagrangian 3-folds in $\mathbb{C}^{3}$.}

We now review the author's three papers $[9,10,11]$ studying special Lagrangian 3 -folds $N$ in $\mathbb{C}^{3}$ invariant under the $\mathrm{U}(1)$-action

$$
\mathrm{e}^{i \theta}:\left(z_{1}, z_{2}, z_{3}\right) \longmapsto\left(\mathrm{e}^{i \theta} z_{1}, \mathrm{e}^{-i \theta} z_{2}, z_{3}\right) \quad \text { for } \mathrm{e}^{i \theta} \in \mathrm{U}(1) \text {. }
$$

The three papers are briefly surveyed in [12]. The results most relevant to this paper are in $[11, \S 8]$, which constructs large families of U(1)-invariant special Lagrangian fibrations on open subsets of $\mathbb{C}^{3}$. These will be summarized in $\S 4.5$, after some introductory material needed to understand and explain them.

\subsection{Background material from analysis.}

A closed, bounded, contractible subset $S$ in $\mathbb{R}^{n}$ will be called a domain if the interior $S^{\circ}$ of $S$ is connected with $S=\overline{S^{\circ}}$, and the boundary $\partial S=S \backslash S^{\circ}$ is a compact embedded hypersurface in $\mathbb{R}^{n}$. A domain $S$ in $\mathbb{R}^{2}$ is called strictly convex if $S$ is convex and the curvature of $\partial S$ is nonzero at every point.

Let $S$ be a domain in $\mathbb{R}^{n}$. Define $C^{k}(S)$ for $k \geqslant 0$ to be the space of continuous functions $f: S \rightarrow \mathbb{R}$ with $k$ continuous derivatives, and define the norm $\|\cdot\|_{C^{k}}$ on $C^{k}(S)$ by $\|f\|_{C^{k}}=\sum_{j=0}^{k} \sup _{S}\left|\partial^{j} f\right|$. Then $C^{k}(S)$ is a 
Banach space. Write $C^{\infty}(S)=\bigcap_{k=0}^{\infty} C^{k}(S)$. For $k \geqslant 0$ and $\alpha \in(0,1)$, define the Hölder space $C^{k, \alpha}(S)$ to be the subset of $f \in C^{k}(S)$ for which

$$
\left[\partial^{k} f\right]_{\alpha}=\sup _{x \neq y \in S} \frac{\left|\partial^{k} f(x)-\partial^{k} f(y)\right|}{|x-y|^{\alpha}}
$$

is finite, and define the Hölder norm on $C^{k, \alpha}(S)$ to be $\|f\|_{C^{k, \alpha}}=\|f\|_{C^{k}}+$ $\left[\partial^{k} f\right]_{\alpha}$. Again, $C^{k, \alpha}(S)$ is a Banach space.

A second-order quasilinear operator $Q: C^{2}(S) \rightarrow C^{0}(S)$ is an operator of the form

$$
(Q u)(x)=\sum_{i, j=1}^{n} a^{i j}(x, u, \partial u) \frac{\partial^{2} u}{\partial x_{i} \partial x_{j}}(x)+b(x, u, \partial u),
$$

where $a^{i j}$ and $b$ are continuous maps $S \times \mathbb{R} \times\left(\mathbb{R}^{n}\right)^{*} \rightarrow \mathbb{R}$, and $a^{i j}=a^{j i}$ for all $i, j=1, \ldots, n$. We call the functions $a^{i j}$ and $b$ the coefficients of $Q$. We call $Q$ elliptic if the symmetric $n \times n$ matrix $\left(a^{i j}\right)$ is positive definite at every point.

A second-order quasilinear operator $Q$ is in divergence form if it is written

$$
(Q u)(x)=\sum_{j=1}^{n} \frac{\partial}{\partial x_{j}}\left(a^{j}(x, u, \partial u)\right)+b(x, u, \partial u)
$$

for functions $a^{j} \in C^{1}\left(S \times \mathbb{R} \times\left(\mathbb{R}^{n}\right)^{*}\right)$ for $j=1, \ldots, n$ and $b \in C^{0}(S \times \mathbb{R} \times$ $\left.\left(\mathbb{R}^{n}\right)^{*}\right)$. If $Q$ is in divergence form, we say that integrable functions $u, f$ are a weak solution of the equation $Q u=f$ if $u$ is weakly differentiable with weak derivative $\partial u$, and $a^{j}(x, u, \partial u), b(x, u, \partial u)$ are integrable with

$$
-\sum_{j=1}^{n} \int_{S} \frac{\partial \psi}{\partial x_{j}} \cdot a^{j}(x, u, \partial u) \mathrm{d} \mathbf{x}+\int_{S} \psi \cdot b(x, u, \partial u) \mathrm{d} \mathbf{x}=\int_{S} \psi \cdot f \mathrm{~d} \mathbf{x}
$$

for all $\psi \in C^{1}(S)$ with $\left.\psi\right|_{\partial S} \equiv 0$.

If $Q$ is a second-order quasilinear operator, we may interpret the equation $Q u=f$ in three different senses:

- We just say that $Q u=f$ if $u \in C^{2}(S), f \in C^{0}(S)$ and $Q u=f$ in $C^{0}(S)$ in the usual way.

- We say that $Q u=f$ holds with weak derivatives if $u$ is twice weakly differentiable and $Q u=f$ holds almost everywhere, defining $Q u$ using weak derivatives. 
- We say that $Q u=f$ holds weakly if $Q$ is in divergence form and $u$ is a weak solution of $Q u=f$. Note that this requires only that $u$ be once weakly differentiable, and the second derivatives of $u$ need not exist even weakly.

Clearly the first sense implies the second, which implies the third. If $Q$ is elliptic and $a^{j}, b, f$ are suitably regular, one can usually show that a weak solution to $Q u=f$ is a classical solution, so that the three senses are equivalent. But for singular equations that are not elliptic at every point, the three senses are distinct.

\subsection{Finding the equations.}

Let $N$ be a special Lagrangian 3 -fold in $\mathbb{C}^{3}$ invariant under the $\mathrm{U}(1)$-action (6). Locally we can write $N$ in the form

$$
\begin{gathered}
N=\left\{\left(z_{1}, z_{2}, z_{3}\right) \in \mathbb{C}^{3}: z_{1} z_{2}=v(x, y)+i y, \quad z_{3}=x+i u(x, y),\right. \\
\left.\left|z_{1}\right|^{2}-\left|z_{2}\right|^{2}=2 a, \quad(x, y) \in S\right\},
\end{gathered}
$$

where $S$ is a domain in $\mathbb{R}^{2}, a \in \mathbb{R}$ and $u, v: S \rightarrow \mathbb{R}$ are continuous.

Here $\left|z_{1}\right|^{2}-\left|z_{2}\right|^{2}$ is the moment map of the $\mathrm{U}(1)$-action (6), and so $\left|z_{1}\right|^{2}-\left|z_{2}\right|^{2}$ is constant on any U(1)-invariant Lagrangian 3-fold in $\mathbb{C}^{3}$. We choose the constant to be $2 a$. Effectively (8) just means that we choose $x=\operatorname{Re}\left(z_{3}\right)$ and $y=\operatorname{Im}\left(z_{1} z_{2}\right)$ as local coordinates on the 2 -manifold $N / \mathrm{U}(1)$. Then we find [9, Prop. 4.1]:

Proposition 4.1. Let $S, a, u, v$ and $N$ be as above. Then

(a) If $a=0$, then $N$ is a (possibly singular) special Lagrangian 3-fold in $\mathbb{C}^{3}$ if $u, v$ are differentiable and satisfy

$$
\frac{\partial u}{\partial x}=\frac{\partial v}{\partial y} \quad \text { and } \quad \frac{\partial v}{\partial x}=-2\left(v^{2}+y^{2}\right)^{1 / 2} \frac{\partial u}{\partial y},
$$

except at points $(x, 0)$ in $S$ with $v(x, 0)=0$, where $u, v$ need not be differentiable. The singular points of $N$ are those of the form $\left(0,0, z_{3}\right)$, where $z_{3}=x+i u(x, 0)$ for $(x, 0) \in S$ with $v(x, 0)=0$.

(b) If $a \neq 0$, then $N$ is a nonsingular special Lagrangian 3 -fold in $\mathbb{C}^{3}$ if and only if $u, v$ are differentiable in $S$ and satisfy

$$
\frac{\partial u}{\partial x}=\frac{\partial v}{\partial y} \quad \text { and } \quad \frac{\partial v}{\partial x}=-2\left(v^{2}+y^{2}+a^{2}\right)^{1 / 2} \frac{\partial u}{\partial y} .
$$


The proof is elementary: at each point $\mathbf{z} \in N$ we calculate the tangent space $T_{\mathbf{z}} N$ in terms of $\partial u, \partial v$, and use Proposition 2.3 to find the conditions for $T_{\mathbf{z}} N$ to be a special Lagrangian $\mathbb{R}^{3}$ in $\mathbb{C}^{3}$. If $\mathbf{z}=\left(0,0, z_{3}\right)$ then $\mathrm{d}\left(\left|z_{1}\right|^{2}-\right.$ $\left.\left|z_{2}\right|^{2}\right)=0$ at $\mathbf{z}$, so $\mathbf{z}$ is a singular point of $N$, and $T_{\mathbf{z}} N$ does not exist.

Using (10) to write $\frac{\partial}{\partial y}\left(\frac{\partial u}{\partial x}\right)$ and $\frac{\partial}{\partial x}\left(\frac{\partial u}{\partial y}\right)$ in terms of $v$ and setting $\frac{\partial^{2} u}{\partial y \partial x}=$ $\frac{\partial^{2} u}{\partial x \partial y}$, we easily prove $[9$, Prop. 8.1$]$ :

Proposition 4.2. Let $S$ be a domain in $\mathbb{R}^{2}$ and $u, v \in C^{2}(S)$ satisfy (10) for $a \neq 0$. Then

$$
\frac{\partial}{\partial x}\left[\left(v^{2}+y^{2}+a^{2}\right)^{-1 / 2} \frac{\partial v}{\partial x}\right]+2 \frac{\partial^{2} v}{\partial y^{2}}=0
$$

Conversely, if $v \in C^{2}(S)$ satisfies (11) then there exists $u \in C^{2}(S)$, unique up to addition of a constant $u \mapsto u+c$, such that $u, v$ satisfy (10).

Now (11) is a second order quasilinear elliptic equation, in divergence form. Thus we can consider weak solutions of (11) when $a=0$, which need be only once weakly differentiable. We shall be interested in solutions of (9) with singularities, and the corresponding SL 3 -folds $N$. It will be helpful to define a class of singular solutions of (9).

Definition 4.3. Let $S$ be a domain in $\mathbb{R}^{2}$ and $u, v \in C^{0}(S)$. We say that $(u, v)$ is a singular solution of $(9)$ if

(i) $u, v$ are weakly differentiable, and their weak derivatives $\frac{\partial u}{\partial x}, \frac{\partial u}{\partial y}, \frac{\partial v}{\partial x}, \frac{\partial v}{\partial y}$ satisfy (9).

(ii) $v$ is a weak solution of (11) with $a=0$, as in $\S 4.2$.

(iii) Define the singular points of $u, v$ to be the $(x, 0) \in S$ with $v(x, 0)=0$. Then except at singular points, $u, v$ are $C^{2}$ in $S$ and real analytic in $S^{\circ}$, and satisfy (9) in the classical sense.

(iv) For $a \in(0,1]$ there exist $u_{a}, v_{a} \in C^{2}(S)$ satisfying (10) such that $u_{a} \rightarrow u$ and $v_{a} \rightarrow v$ in $C^{0}(S)$ as $a \rightarrow 0_{+}$.

This list of properties is somewhat arbitrary. The point is that $[10, \S 8-$ $\S 9]$ gives powerful existence and uniqueness results for solutions $u, v$ of (9) satisfying conditions (i)-(iv) and various boundary conditions on $\partial S$, and all of (i)-(iv) are useful in different contexts. 


\subsection{Examples.}

The following example is due to Harvey and Lawson [7, §III.3.A].

Example 4.4. The map $f: \mathbb{C}^{3} \rightarrow \mathbb{R}^{3}$ defined by

$$
f:\left(z_{1}, z_{2}, z_{3}\right) \longmapsto\left(\left|z_{1}\right|^{2}-\left|z_{2}\right|^{2},\left|z_{1}\right|^{2}-\left|z_{3}\right|^{2}, \operatorname{Im}\left(z_{1} z_{2} z_{3}\right)\right)
$$

is a smooth special Lagrangian fibration of $\mathbb{C}^{3}$. The fibres of $f$ are invariant under a subgroup $\mathrm{U}(1)^{2}$ in $\mathrm{SU}(3)$ acting by

$$
\left(\mathrm{e}^{i \theta_{1}}, \mathrm{e}^{i \theta_{2}}\right):\left(z_{1}, z_{2}, z_{3}\right) \longmapsto\left(\mathrm{e}^{i \theta_{1}} z_{1}, \mathrm{e}^{i \theta_{2}} z_{2}, \mathrm{e}^{-i\left(\theta_{1}+\theta_{2}\right)} z_{3}\right),
$$

and every $\mathrm{U}(1)^{2}$-invariant SL 3 -fold in $\mathbb{C}^{3}$ is locally made up of fibres of $f$.

Calculation shows that the discriminant of $f$, in the sense of $\S 3$, is

$$
\Delta=\{(\alpha, \alpha, 0),(0,-\alpha, 0),(0,0,-\alpha): \alpha \geqslant 0\} \subset \mathbb{R}^{3} .
$$

It is a trivalent graph, of codimension two in $\mathbb{R}^{3}$.

We are interested in a family of particular fibres of $f$ which decompose into two pieces. Let $a \in \mathbb{R}$, and define

$$
\begin{gathered}
N_{a}=\left\{\left(z_{1}, z_{2}, z_{3}\right) \in \mathbb{C}^{3}:\left|z_{1}\right|^{2}-a=\left|z_{2}\right|^{2}+a=\left|z_{3}\right|^{2}+|a|,\right. \\
\left.\operatorname{Im}\left(z_{1} z_{2} z_{3}\right)=0, \quad \operatorname{Re}\left(z_{1} z_{2} z_{3}\right) \geqslant 0\right\} .
\end{gathered}
$$

Then $N_{a}$ is half of the fibre $f^{-1}(2 a, 2 a, 0)$ when $a \geqslant 0$, and half of the fibre $f^{-1}(2 a, 0,0)$ when $a<0$, so $N_{a}$ is special Lagrangian.

One can show that $N_{a}$ is a nonsingular SL 3 -fold diffeomorphic to $\mathcal{S}^{1} \times \mathbb{R}^{2}$ when $a \neq 0$, and $N_{0}$ is an SL $T^{2}$-cone with one singular point at $(0,0,0)$. Note that even though $N_{a}$ is defined using an inequality $\operatorname{Re}\left(z_{1} z_{2} z_{3}\right) \geqslant 0$, it has no boundary. This is because the fibres $f^{-1}(2 a, 2 a, 0)$ for $a>0$, and $f^{-1}(2 a, 0,0)$ for $a<0$, are actually the union of two nonsingular SL 3-folds $\mathcal{S}^{1} \times \mathbb{R}^{2}$, which intersect in a circle. The inequality is used to pick out one of these two SL 3-folds.

By [9, Th. 5.1], these SL 3-folds $N_{a}$ can be written in the form (8).

Theorem 4.5. Let $a \in \mathbb{R}$. Then there exist unique $u_{a}, v_{a}: \mathbb{R}^{2} \rightarrow \mathbb{R}$ such that

$$
\begin{aligned}
N=\left\{\left(z_{1}, z_{2}, z_{3}\right) \in \mathbb{C}^{3}: \operatorname{Im}\left(z_{3}\right)=u_{a}\left(\operatorname{Re}\left(z_{3}\right), \operatorname{Im}\left(z_{1} z_{2}\right)\right),\right. & \\
\operatorname{Re}\left(z_{1} z_{2}\right) & \left.=v_{a}\left(\operatorname{Re}\left(z_{3}\right), \operatorname{Im}\left(z_{1} z_{2}\right)\right), \quad\left|z_{1}\right|^{2}-\left|z_{2}\right|^{2}=2 a\right\}
\end{aligned}
$$

is the special Lagrangian 3-fold $N_{a}$ of (15). Furthermore: 
(a) $u_{a}, v_{a}$ are smooth on $\mathbb{R}^{2}$ and satisfy $(10)$, except at $(0,0)$ when $a=0$, where they are only continuous.

(b) $u_{a}(x, y)<0$ when $y>0$ for all $x$, and $u_{a}(x, 0)=0$ for all $x$, and $u_{a}(x, y)>0$ when $y<0$ for all $x$.

(c) $v_{a}(x, y)>0$ when $x>0$ for all $y$, and $v_{a}(0, y)=0$ for all $y$, and $v_{a}(x, y)<0$ when $x<0$ for all $y$.

(d) $u_{a}(0, y)=-y\left(|a|+\sqrt{y^{2}+a^{2}}\right)^{-1 / 2}$ for all $y$.

(e) $v_{a}(x, 0)=x\left(x^{2}+2|a|\right)^{1 / 2}$ for all $x$.

(f) $u_{-a} \equiv u_{a}$ and $v_{-a} \equiv v_{a}$.

In fact $[9, \S 5]$ considers only the case $a \geqslant 0$, but the case $a<0$ and part (f) follow quickly by exchanging $z_{1}$ and $z_{2}$. Note that although the $N_{a}$ for $a>0$ and $a<0$ are both diffeomorphic to $\mathcal{S}^{1} \times \mathbb{R}^{2}$, nonetheless there is a topological change as $a$ goes from positive to negative, as the fibres undergo a surgery, a Dehn twist on $\mathcal{S}^{1}$.

\subsection{Generating $u, v$ from a potential $f$.}

In [9, Prop. 7.1] we show that solutions $u, v \in C^{1}(S)$ of (10) come from a potential $f \in C^{2}(S)$ with $\frac{\partial f}{\partial y}=u$ and $\frac{\partial f}{\partial x}=v$.

Proposition 4.6. Let $S$ be a domain in $\mathbb{R}^{2}$ and $u, v \in C^{1}(S)$ satisfy (10) for $a \neq 0$. Then there exists $f \in C^{2}(S)$ with $\frac{\partial f}{\partial y}=u, \frac{\partial f}{\partial x}=v$ and

$$
\left(\left(\frac{\partial f}{\partial x}\right)^{2}+y^{2}+a^{2}\right)^{-1 / 2} \frac{\partial^{2} f}{\partial x^{2}}+2 \frac{\partial^{2} f}{\partial y^{2}}=0
$$

This $f$ is unique up to addition of a constant, $f \mapsto f+c$. Conversely, all solutions of (17) yield solutions of (10).

Equation (17) is a second-order quasilinear elliptic equation, singular when $a=0$, which may be written in divergence form. The following condensation of [9, Th. 7.6] and [10, Th.s $9.20 \& 9.21]$ proves existence and uniqueness for the Dirichlet problem for (17).

Theorem 4.7. Suppose $S$ is a strictly convex domain in $\mathbb{R}^{2}$ invariant under $(x, y) \mapsto(x,-y)$, and $k \geqslant 0, \alpha \in(0,1)$. Let $a \in \mathbb{R}$ and $\phi \in C^{k+3, \alpha}(\partial S)$. Then if $a \neq 0$ there exists a unique $f \in C^{k+3, \alpha}(S)$ with $\left.f\right|_{\partial S}=\phi$ satisfying 
(17). If $a=0$ there exists a unique $f \in C^{1}(S)$ with $\left.f\right|_{\partial S}=\phi$, which is twice weakly differentiable and satisfies (17) with weak derivatives.

Define $u=\frac{\partial f}{\partial y}$ and $v=\frac{\partial f}{\partial x}$. If $a \neq 0$ then $u, v \in C^{k+2, \alpha}(S)$ satisfy (10), and if $a=0$ then $u, v \in C^{0}(S)$ are a singular solution of (9), in the sense of Definition 4.3. Furthermore, $f$ depends continuously in $C^{1}(S)$, and $u, v$ depend continuously in $C^{0}(S)$, on $(\phi, a)$ in $C^{k+3, \alpha}(\partial S) \times \mathbb{R}$.

Combining Proposition 4.1 and Theorem 4.7 gives existence and uniqueness for a large class of $\mathrm{U}(1)$-invariant SL 3 -folds in $\mathbb{C}^{3}$, with boundary conditions, including singular SL 3 -folds. It is interesting that this existence and uniqueness is entirely unaffected by singularities appearing in $S^{\circ}$.

\subsection{Special Lagrangian fibrations.}

We can use Theorem 4.7 to construct large families of special Lagrangian fibrations of open subsets of $\mathbb{C}^{3}$ invariant under the U(1)-action (6), including singular fibres.

Definition 4.8. Let $S$ be a strictly convex domain in $\mathbb{R}^{2}$ invariant under $(x, y) \mapsto(x,-y)$, let $U$ be an open set in $\mathbb{R}^{3}$, and $\alpha \in(0,1)$. Suppose $\Phi: U \rightarrow C^{3, \alpha}(\partial S)$ is a continuous map such that if $(a, b, c) \neq\left(a, b^{\prime}, c^{\prime}\right)$ in $U$ then $\Phi(a, b, c)-\Phi\left(a, b^{\prime}, c^{\prime}\right)$ has exactly one local maximum and one local minimum in $\partial S$.

Let $\boldsymbol{\alpha}=(a, b, c) \in U$, and let $f_{\boldsymbol{\alpha}} \in C^{3, \alpha}(S)$ be the unique (weak) solution of (17) with $\left.f_{\boldsymbol{\alpha}}\right|_{\partial S}=\Phi(\boldsymbol{\alpha})$, which exists by Theorem 4.7. Define $u_{\boldsymbol{\alpha}}=\frac{\partial f_{\boldsymbol{\alpha}}}{\partial y}$ and $v_{\boldsymbol{\alpha}}=\frac{\partial f_{\boldsymbol{\alpha}}}{\partial x}$. Then $\left(u_{\boldsymbol{\alpha}}, v_{\boldsymbol{\alpha}}\right)$ is a solution of (10) if $a \neq 0$, and a singular solution of (9) if $a=0$. Also $u_{\boldsymbol{\alpha}}, v_{\boldsymbol{\alpha}}$ depend continuously on $\boldsymbol{\alpha} \in U$ in $C^{0}(S)$, by Theorem 4.7 .

For each $\boldsymbol{\alpha}=(a, b, c)$ in $U$, define $N_{\boldsymbol{\alpha}}$ in $\mathbb{C}^{3}$ by

$$
\begin{gathered}
N_{\boldsymbol{\alpha}}=\left\{\left(z_{1}, z_{2}, z_{3}\right) \in \mathbb{C}^{3}: z_{1} z_{2}=v_{\boldsymbol{\alpha}}(x, y)+i y, \quad z_{3}=x+i u_{\boldsymbol{\alpha}}(x, y),\right. \\
\left.\left|z_{1}\right|^{2}-\left|z_{2}\right|^{2}=2 a, \quad(x, y) \in S^{\circ}\right\} .
\end{gathered}
$$

Then $N_{\boldsymbol{\alpha}}$ is a noncompact SL 3 -fold without boundary in $\mathbb{C}^{3}$, which is nonsingular if $a \neq 0$, by Proposition 4.1.

By [11, Th. 8.2] the $N_{\boldsymbol{\alpha}}$ are the fibres of a special Lagrangian fibration.

Theorem 4.9. In the situation of Definition 4.8, if $\boldsymbol{\alpha} \neq \boldsymbol{\alpha}^{\prime}$ in $U$ then $N_{\boldsymbol{\alpha}} \cap N_{\boldsymbol{\alpha}^{\prime}}=\emptyset$. There exists an open set $V \subset \mathbb{C}^{3}$ and a continuous, surjective map $F: V \rightarrow U$ such that $F^{-1}(\boldsymbol{\alpha})=N_{\boldsymbol{\alpha}}$ for all $\boldsymbol{\alpha} \in U$. Thus, $F$ is a special Lagrangian fibration of $V \subset \mathbb{C}^{3}$, which may include singular fibres. 
The main step in the proof is to show that distinct $N_{\boldsymbol{\alpha}}$ do not intersect, so that they fibre $V=\bigcup_{\boldsymbol{\alpha} \in U} N_{\boldsymbol{\alpha}}$. The tool we use to do this is the following result $[9$, Th. 7.11$],[11$, Th. 7.10$]$ :

Theorem 4.10. Suppose $S$ is a strictly convex domain in $\mathbb{R}^{2}$ invariant un$\operatorname{der}(x, y) \mapsto(x,-y)$, and $a \in \mathbb{R}, k \geqslant 0, \alpha \in(0,1)$, and $\phi_{1}, \phi_{2} \in C^{k+3, \alpha}(\partial S)$. Let $u_{j}, v_{j} \in C^{0}(S)$ be the (singular) solution of (9) or (10) constructed in Theorem 4.7 from $\phi_{j}$, for $j=1,2$.

Suppose $\phi_{1}-\phi_{2}$ has l local maxima and l local minima on $\partial S$. Then $\left(u_{1}, v_{1}\right)-\left(u_{2}, v_{2}\right)$ has finitely many zeroes in $S^{\circ}$, all isolated. Let there be $n$ zeroes in $S^{\circ}$ with multiplicities $k_{1}, \ldots, k_{n}$. Then $\sum_{i=1}^{n} k_{i} \leqslant l-1$.

Here isolated zeroes of $\left(u_{1}, v_{1}\right)-\left(u_{2}, v_{2}\right)$ have a multiplicity, defined in [11, Def. 7.1], which is a positive integer by $[9, \S 6.1]$ and [11, Cor. 7.6]. The result provides an upper bound for the number of zeroes of $\left(u_{1}, v_{2}\right)-\left(u_{2}, v_{2}\right)$ in $S^{\circ}$, counted with multiplicity, in terms of the boundary data $\phi_{1}, \phi_{2}$.

Suppose $\alpha=(a, b, c)$ and $\alpha^{\prime}=\left(a^{\prime}, b^{\prime}, c^{\prime}\right)$ are distinct elements of $U$. If $a \neq a^{\prime}$ then $N_{\boldsymbol{\alpha}} \cap N_{\boldsymbol{\alpha}^{\prime}}=\emptyset$, since $\left|z_{1}\right|^{2}-\left|z_{2}\right|^{2}$ is $2 a$ on $N_{\boldsymbol{\alpha}}$ and $2 a^{\prime}$ on $N_{\boldsymbol{\alpha}^{\prime}}$. If $a=a^{\prime}$ then $\Phi(\boldsymbol{\alpha})-\Phi\left(\boldsymbol{\alpha}^{\prime}\right)$ has one local maximum and one local minimum in $\partial S$, by Definition 4.8. So Theorem 4.10 applies with $l=1$ to show that $\left(u_{\boldsymbol{\alpha}}, v_{\boldsymbol{\alpha}}\right)-\left(u_{\boldsymbol{\alpha}^{\prime}}, v_{\boldsymbol{\alpha}^{\prime}}\right)$ has no zeroes in $S^{\circ}$, and again $N_{\boldsymbol{\alpha}} \cap N_{\boldsymbol{\alpha}^{\prime}}=\emptyset$. Thus distinct $N_{\boldsymbol{\alpha}}$ do not intersect.

For reasons explained in $[11, \S 8]$, we chose to define $N_{\boldsymbol{\alpha}}$ in (18) over $S^{\circ}$ rather than $S$, and so end up with a noncompact SL 3 -fold without boundary rather than a compact SL 3-fold with boundary. The results can be extended to compact SL 3-folds $\bar{N}_{\boldsymbol{\alpha}}$ with boundary, but it makes the statements rather more complicated, and introduces new technical problems when $\bar{N}_{\boldsymbol{\alpha}}$ has singularities on its boundary.

There is a simple way [11, Ex. 8.3] to produce families $\Phi$ satisfying Definition 4.8 , and thus generate many SL fibrations of open subsets of $\mathbb{C}^{3}$.

Example 4.11. Let $S$ be a strictly convex domain in $\mathbb{R}^{2}$ invariant under $(x, y) \mapsto(x,-y)$, let $\alpha \in(0,1)$ and $\phi \in C^{3, \alpha}(\partial S)$. Define $U=\mathbb{R}^{3}$ and $\Phi: \mathbb{R}^{3} \rightarrow C^{3, \alpha}(\partial S)$ by $\Phi(a, b, c)=\phi+b x+c y$. If $(a, b, c) \neq\left(a, b^{\prime}, c^{\prime}\right)$ then $\Phi(a, b, c)-\Phi\left(a, b^{\prime}, c^{\prime}\right)=\left(b-b^{\prime}\right) x+\left(c-c^{\prime}\right) y \in C^{\infty}(\partial S)$. As $b-b^{\prime}, c-c^{\prime}$ are not both zero and $S$ is strictly convex, it easily follows that $\left(b-b^{\prime}\right) x+\left(c-c^{\prime}\right) y$ has one local maximum and one local minimum in $\partial S$.

Hence the conditions of Definition 4.8 hold for $S, U$ and $\Phi$, and so Theorem 4.9 defines an open set $V \subset \mathbb{C}^{3}$ and a special Lagrangian fibration $F: V \rightarrow \mathbb{C}^{3}$. One can also show that changing the parameter $c$ in 
$U=\mathbb{R}^{3}$ just translates the fibres $N_{\boldsymbol{\alpha}}$ in $\mathbb{C}^{3}$, and $V=\left\{\left(z_{1}, z_{2}, z_{3}\right) \in \mathbb{C}^{3}\right.$ : $\left.\left(\operatorname{Re} z_{3}, \operatorname{Im} z_{1} z_{2}\right) \in S^{\circ}\right\}$.

\subsection{A rough classification of singular points.}

In $[11, \S 9]$ we study singular points of a singular solution $u, v$ of $(9)$.

Definition 4.12. Let $S$ be a domain in $\mathbb{R}^{2}$, and $u, v \in C^{0}(S)$ a singular solution of (9), as in Definition 4.3. Suppose for simplicity that $S$ is invariant under $(x, y) \mapsto(x,-y)$. Define $u^{\prime}, v^{\prime} \in C^{0}(S)$ by $u^{\prime}(x, y)=u(x,-y)$ and $v^{\prime}(x, y)=-v(x,-y)$. Then $u^{\prime}, v^{\prime}$ is also a singular solution of $(9)$.

A singular point, or singularity, of $(u, v)$ is a point $(b, 0) \in S$ with $v(b, 0)=0$. Observe that a singularity of $(u, v)$ is automatically a zero of $(u, v)-\left(u^{\prime}, v^{\prime}\right)$. Conversely, a zero of $(u, v)-\left(u^{\prime}, v^{\prime}\right)$ on the $x$-axis is a singularity. A singularity of $(u, v)$ is called isolated if it is an isolated zero of $(u, v)-\left(u^{\prime}, v^{\prime}\right)$.

Define the multiplicity of an isolated singularity $(b, 0)$ of $(u, v)$ in $S^{\circ}$ to be the winding number of $(u, v)-\left(u^{\prime}, v^{\prime}\right)$ about 0 along the positively oriented circle $\gamma_{\epsilon}(b, 0)$ of radius $\epsilon$ about $(b, 0)$, where $\epsilon>0$ is chosen small enough that $\gamma_{\epsilon}(b, 0)$ lies in $S^{\circ}$ and $(b, 0)$ is the only zero of $(u, v)-\left(u^{\prime}, v^{\prime}\right)$ inside or on $\gamma_{\epsilon}(b, 0)$. By [11, Cor. 7.6], this multiplicity is a positive integer.

Under mild conditions the singularities in $S^{\circ}$ are isolated, [11, Th. 9.2]:

Theorem 4.13. Let $S$ be a domain in $\mathbb{R}^{2}$ invariant under $(x, y) \mapsto(x,-y)$, and $u, v \in C^{0}(S)$ a singular solution of $(9)$. If $u(x,-y) \equiv u(x, y)$ and $v(x,-y) \equiv-v(x, y)$ then $(u, v)$ is singular along the $x$-axis in $S$, and the singularities are nonisolated. Otherwise there are at most countably many singularities of $(u, v)$ in $S^{\circ}$, all isolated.

We divide isolated singularities $(b, 0)$ into four types, depending on the behaviour of $v(x, 0)$ near $(b, 0)$.

Definition 4.14. Let $S$ be a domain in $\mathbb{R}^{2}$, and $u, v \in C^{0}(S)$ a singular solution of $(9)$, as in Definition 4.3. Suppose $(b, 0)$ is an isolated singular point of $(u, v)$ in $S^{\circ}$. Then there exists $\epsilon>0$ such that for $0<|x-b|<\epsilon$ we have $(x, 0) \in S^{\circ}$ and $v(x, 0) \neq 0$. So by continuity $v$ is either positive or negative on each of $(b-\epsilon, b) \times\{0\}$ and $(b, b+\epsilon) \times\{0\}$.

(i) if $v(x)<0$ for $x \in(b-\epsilon, b)$ and $v(x)>0$ for $x \in(b, b+\epsilon)$ we say the singularity $(b, 0)$ is of increasing type. 
(ii) if $v(x)>0$ for $x \in(b-\epsilon, b)$ and $v(x)<0$ for $x \in(b, b+\epsilon)$ we say the singularity $(b, 0)$ is of decreasing type.

(iii) if $v(x)<0$ for $x \in(b-\epsilon, b)$ and $v(x)<0$ for $x \in(b, b+\epsilon)$ we say the singularity $(b, 0)$ is of maximum type.

(iv) if $v(x)>0$ for $x \in(b-\epsilon, b)$ and $v(x)>0$ for $x \in(b, b+\epsilon)$ we say the singularity $(b, 0)$ is of minimum type.

The type determines if the multiplicity is even or odd, [11, Prop. 9.4].

Proposition 4.15. Let $u, v \in C^{0}(S)$ be a singular solution of (9) on a domain $S$ in $\mathbb{R}^{2}$, and $(b, 0)$ be an isolated singularity of $(u, v)$ in $S^{\circ}$ with multiplicity $k$. If $(b, 0)$ is of increasing or decreasing type then $k$ is odd, and if $(b, 0)$ is of maximum or minimum type then $k$ is even.

Theorem 4.10 gives a criterion for finitely many singularities, [11, Th. 9.7]:

Theorem 4.16. Suppose $S$ is a strictly convex domain in $\mathbb{R}^{2}$ invariant un$\operatorname{der}(x, y) \mapsto(x,-y)$, and $\phi \in C^{k+3, \alpha}(\partial S)$ for $k \geqslant 0$ and $\alpha \in(0,1)$. Let $u, v$ be the singular solution of $(9)$ in $C^{0}(S)$ constructed from $\phi$ in Theorem 4.7 .

Define $\phi^{\prime} \in C^{k+3, \alpha}(\partial S)$ by $\phi^{\prime}(x, y)=-\phi(x,-y)$. Suppose $\phi-\phi^{\prime}$ has $l$ local maxima and $l$ local minima on $\partial S$. Then $(u, v)$ has finitely many singularities in $S^{\circ}$. Let there be $n$ singularities in $S^{\circ}$ with multiplicities $k_{1}, \ldots, k_{n}$. Then $\sum_{i=1}^{n} k_{i} \leqslant l-1$.

By applying Theorem 4.7 with $S$ the unit disc in $\mathbb{R}^{2}$ and $\phi$ a linear combination of functions $\sin (j \theta), \cos (j \theta)$ on the unit circle $\partial S$, we show [11, Cor. 10.10]:

Theorem 4.17. There exist examples of singular solutions $u, v$ of (9) with isolated singularities of every possible multiplicity $n \geqslant 1$, and with both possible types allowed by Proposition 4.15.

Combining this with Proposition 4.1 gives examples of SL 3 -folds in $\mathbb{C}^{3}$ with singularities of an infinite number of different geometrical/topological types. We also show in $[11, \S 10.4]$ that singular points with multiplicity $n \geqslant 1$ occur in real codimension $n$ in the family of all SL 3-folds invariant under the U(1)-action (6), in a well-defined sense. 


\section{Two model special Lagrangian fibrations.}

We shall now define two piecewise smooth special Lagrangian fibrations $F, F^{\prime}: \mathbb{C}^{3} \rightarrow \mathbb{R}^{3}$ with singular fibres of codimension one in $\mathbb{R}^{3}$. These will be our local models for the most generic kind of singularity in special Lagrangian fibrations of generic Calabi-Yau 3-folds. Here is the first.

Theorem 5.1. For each $a \in \mathbb{R}$ and $c \in \mathbb{C}$, define $N_{a, c}$ in $\mathbb{C}^{3}$ by

$$
\begin{gathered}
N_{a, c}=\left\{\left(z_{1}, z_{2}, z_{3}\right) \in \mathbb{C}^{3}:\left|z_{1}\right|^{2}-a=\left|z_{2}\right|^{2}+a=\left|z_{3}-c\right|^{2}+|a|,\right. \\
\left.\operatorname{Im}\left(z_{1} z_{2}\left(z_{3}-c\right)\right)=0, \quad \operatorname{Re}\left(z_{1} z_{2}\left(z_{3}-c\right)\right) \geqslant 0\right\} .
\end{gathered}
$$

Then $N_{a, c}$ is a nonsingular $S L 3$-fold diffeomorphic to $\mathcal{S}^{1} \times \mathbb{R}^{2}$ if $a \neq 0$, and $N_{0, c}$ is an $S L T^{2}$-cone singular at $(0,0, c)$. Define $F: \mathbb{C}^{3} \rightarrow \mathbb{R} \times \mathbb{C}$ by

$$
\begin{gathered}
F\left(z_{1}, z_{2}, z_{3}\right)=(a, b), \quad \text { where } \quad 2 a=\left|z_{1}\right|^{2}-\left|z_{2}\right|^{2} \\
\text { and } \quad b= \begin{cases}z_{3}, & a=z_{1}=z_{2}=0, \\
z_{3}-\bar{z}_{1} \bar{z}_{2} /\left|z_{1}\right|, & a \geqslant 0, \quad z_{1} \neq 0 \\
z_{3}-\bar{z}_{1} \bar{z}_{2} /\left|z_{2}\right|, & a<0 .\end{cases}
\end{gathered}
$$

Then $F^{-1}(a, c)=N_{a, c}$ for all $a, c \in \mathbb{R} \times \mathbb{C}$, and $F$ is a continuous, piecewisesmooth $S L$ fibration of $\mathbb{C}^{3}$, which is not smooth on $\left|z_{1}\right|=\left|z_{2}\right|$.

Proof. Comparing (15) and (19) shows that $N_{a, c}$ is the translation by $(0,0, c)$ of the SL 3 -fold $N_{a}$ of $\S 4.3$. Hence $N_{a, c}$ is a nonsingular special Lagrangian $\mathcal{S}^{1} \times \mathbb{R}^{2}$ if $a \neq 0$, and an SL $T^{2}$-cone singular at $(0,0, c)$ if $a=0$. It is also easy to see that $F$ is well-defined, continuous, piecewise smooth, and not smooth on $\left|z_{1}\right|=\left|z_{2}\right|$.

One can show from (19) that if $\left(z_{1}, z_{2}, z_{3}\right) \in N_{a, c}$ then $2 a=\left|z_{1}\right|^{2}-\left|z_{2}\right|^{2}$ and

$$
z_{1} z_{2}\left(z_{3}-c\right)= \begin{cases}\left|z_{1}\right|\left|z_{2}\right|^{2}, & a \geqslant 0 \\ \left|z_{1}\right|^{2}\left|z_{2}\right|, & a<0 .\end{cases}
$$

Thus, if $z_{1} z_{2} \neq 0$ dividing by $z_{1} z_{2}$ and rearranging yields

$$
c= \begin{cases}z_{3}-\left|z_{1}\right|\left|z_{2}\right|^{2} /\left(z_{1} z_{2}\right), & a \geqslant 0, \\ z_{3}-\left|z_{1}\right|^{2}\left|z_{2}\right| /\left(z_{1} z_{2}\right), & a<0 .\end{cases}
$$

Using the equations $\left|z_{1}\right|^{2}=z_{1} \bar{z}_{1}$ and $\left|z_{2}\right|^{2}=z_{2} \bar{z}_{2}$ to rewrite these expressions gives the second case of $(21)$ when $z_{2} \neq 0$ and the third when $z_{1} \neq 0$. 
If $z_{1} z_{2}=0$, equation (19) implies that $\left|z_{3}-c\right|^{2}=0$, so $c=z_{3}$, giving the first case of (21), the second when $z_{2}=0$ and the third when $z_{1}=0$. So, if $\left(z_{1}, z_{2}, z_{3}\right) \in N_{a, c}$ then we can recover $a, c$ from $\left(z_{1}, z_{2}, z_{3}\right)$ as in (20)-(21). Conversely, for any $\left(z_{1}, z_{2}, z_{3}\right)$ in $\mathbb{C}^{3}$, defining $a, c$ by (20)-(21) and reversing the proof above, we find that $\left(z_{1}, z_{2}, z_{3}\right) \in N_{a, c}$. Hence $F^{-1}(a, c)=N_{a, c}$, and $F$ is a special Lagrangian fibration of $\mathbb{C}^{3}$.

Using Theorem 4.5 we write the fibres $N_{a, c}$ of $F$ in the form (8).

Proposition 5.2. The $S L 3$-folds $N_{a, c}$ of Theorem 5.1 may be written

$$
\begin{aligned}
N_{a, c}=\left\{\left(z_{1}, z_{2}, z_{3}\right)\right. & \in \mathbb{C}^{3}: \operatorname{Im}\left(z_{3}\right)=u_{a, c}\left(\operatorname{Re}\left(z_{3}\right), \operatorname{Im}\left(z_{1} z_{2}\right)\right), \\
\operatorname{Re}\left(z_{1} z_{2}\right) & \left.=v_{a, c}\left(\operatorname{Re}\left(z_{3}\right), \operatorname{Im}\left(z_{1} z_{2}\right)\right), \quad\left|z_{1}\right|^{2}-\left|z_{2}\right|^{2}=2 a\right\}
\end{aligned}
$$

for $u_{a, c}, v_{a, c}: \mathbb{R}^{2} \rightarrow \mathbb{R}$ defined using the functions $u_{a}, v_{a}$ of Theorem 4.5 by

$$
u_{a, c}(x, y)=u_{a}(x-\operatorname{Re} c, y)+\operatorname{Im} c \quad \text { and } \quad v_{a, c}(x, y)=v_{a}(x-\operatorname{Re} c, y) .
$$

By applying the involution $\left(z_{1}, z_{2}, z_{3}\right) \mapsto\left(-z_{1}, z_{2}, z_{3}\right)$ to $\mathbb{C}^{3}$ we transform $F$ to a second SL fibration $F^{\prime}$. The previous two results quickly yield:

Theorem 5.3. For each $a \in \mathbb{R}$ and $c \in \mathbb{C}$, define $N_{a, c}^{\prime}$ in $\mathbb{C}^{3}$ by

$$
\begin{gathered}
N_{a, c}^{\prime}=\left\{\left(z_{1}, z_{2}, z_{3}\right) \in \mathbb{C}^{3}:\left|z_{1}\right|^{2}-a=\left|z_{2}\right|^{2}+a=\left|z_{3}-c\right|^{2}+|a|,\right. \\
\left.\operatorname{Im}\left(z_{1} z_{2}\left(z_{3}-c\right)\right)=0, \quad \operatorname{Re}\left(z_{1} z_{2}\left(z_{3}-c\right)\right) \leqslant 0\right\} .
\end{gathered}
$$

Then $N_{a, c}^{\prime}$ is a nonsingular $S L$ 3-fold diffeomorphic to $\mathcal{S}^{1} \times \mathbb{R}^{2}$ if $a \neq 0$, and $N_{0, c}^{\prime}$ is an $S L T^{2}$-cone singular at $(0,0, c)$. Define $F^{\prime}: \mathbb{C}^{3} \rightarrow \mathbb{R} \times \mathbb{C}$ by

$$
\begin{gathered}
F^{\prime}\left(z_{1}, z_{2}, z_{3}\right)=(a, b), \quad \text { where } \quad 2 a=\left|z_{1}\right|^{2}-\left|z_{2}\right|^{2} \\
\text { and } \quad b= \begin{cases}z_{3}, & a=z_{1}=z_{2}=0, \\
z_{3}+\bar{z}_{1} \bar{z}_{2} /\left|z_{1}\right|, & a \geqslant 0, \quad z_{1} \neq 0 \\
z_{3}+\bar{z}_{1} \bar{z}_{2} /\left|z_{2}\right|, & a<0 .\end{cases}
\end{gathered}
$$

Then $\left(F^{\prime}\right)^{-1}(a, c)=N_{a, c}^{\prime}$ for all $a, c \in \mathbb{R} \times \mathbb{C}$, and $F^{\prime}$ is a continuous, piecewise-smooth SL fibration of $\mathbb{C}^{3}$, which is not smooth on $\left|z_{1}\right|=\left|z_{2}\right|$. 
Proposition 5.4. The $S L 3$-folds $N_{a, c}^{\prime}$ of Theorem 5.3 may be written

$$
\begin{aligned}
N_{a, c}^{\prime}=\left\{\left(z_{1}, z_{2}, z_{3}\right)\right. & \in \mathbb{C}^{3}: \operatorname{Im}\left(z_{3}\right)=u_{a, c}^{\prime}\left(\operatorname{Re}\left(z_{3}\right), \operatorname{Im}\left(z_{1} z_{2}\right)\right) \\
\operatorname{Re}\left(z_{1} z_{2}\right) & \left.=v_{a, c}^{\prime}\left(\operatorname{Re}\left(z_{3}\right), \operatorname{Im}\left(z_{1} z_{2}\right)\right), \quad\left|z_{1}\right|^{2}-\left|z_{2}\right|^{2}=2 a\right\}
\end{aligned}
$$

for $u_{a, c}^{\prime}, v_{a, c}^{\prime}: \mathbb{R}^{2} \rightarrow \mathbb{R}$ defined using the functions $u_{a}, v_{a}$ of Theorem 4.5 by

$$
u_{a, c}^{\prime}(x, y)=-u_{a}(x-\operatorname{Re} c, y)+\operatorname{Im} c \text { and } v_{a, c}^{\prime}(x, y)=-v_{a}(x-\operatorname{Re} c, y) .
$$

\subsection{Discussion.}

Theorems 5.1 and 5.3 define SL fibrations $F, F^{\prime}$ of $\mathbb{C}^{3}$ in which every fibre is invariant under the $\mathrm{U}(1)$-action (6) and is written in the form (8). The singular fibres of the fibration are $N_{0, c}$ for $c \in \mathbb{C}$, which is singular only at $(0,0, c)$. Thus the set of singular points of singular fibres of the fibration is $\{(0,0, c): c \in \mathbb{C}\}$, the complex $z_{3}$-axis.

However, $F$ is not smooth on the whole real hypersurface $\left|z_{1}\right|=\left|z_{2}\right|$, which includes the set of singular points but many other points as well. Thus $F$ fails to be smooth not only at singular points of singular fibres, but also at nonsingular points of singular fibres. We should understand the non-smoothness of $F$ as being related not to a singularity at the point in question, but to a change in the global topology of the whole fibre.

In Theorems 5.1 and 5.3 the base space $B$ is $\mathbb{R} \times \mathbb{C}$, and the discriminant $\Delta$ of Definition 3.2 is $\{0\} \times \mathbb{C}$. Thus, $\Delta$ is of real codimension one in $B$. Now by Proposition 3.5, for smooth SL fibrations $\Delta$ has Hausdorff codimension two in $B$. Therefore the piecewise-smooth SL fibrations of Theorems 5.1 and 5.3 have very different behaviour to smooth SL fibrations.

We can discuss the singular fibres of $F, F^{\prime}$ from the point of view of $\S 4.6$. Observe that $\left(u_{0}, v_{0}\right)$ of Theorem 4.5 is a singular solution of $(9)$ in the sense of Definition 4.3, with an isolated singularity at $(0,0)$. Using parts (b) and (c) of Theorem 4.5, it is easy to show that this singularity is of multiplicity one and increasing type, in the sense of $\S 4.6$.

Now Propositions 5.2 and 5.4 define the singular solutions $\left(u_{0, c}, v_{0, c}\right)$ and $\left(u_{0, c}^{\prime}, v_{0, c}^{\prime}\right)$ for $c \in \mathbb{C}$ in terms of $\left(u_{0}, v_{0}\right)$. It easily follows that $\left(u_{0, c}, v_{0, c}\right)$ has an isolated singularity at $(\operatorname{Re} c, 0)$ of multiplicity one and increasing type, and $\left(u_{0, c}^{\prime}, v_{0, c}^{\prime}\right)$ has an isolated singularity at $(\operatorname{Re} c, 0)$ of multiplicity one and decreasing type. So, the singularities of the fibration $F$ have multiplicity one 
and increasing type, and those of $F^{\prime}$ have multiplicity one and decreasing type, in the sense of $\S 4.6$.

We can also discuss the fibrations using the framework of $\S 2.3$. The singular fibres of $F$ and $F^{\prime}$ are special Lagrangian $T^{2}$-cones modelled on

$$
C=\left\{\left(z_{1}, z_{2}, z_{3}\right):\left|z_{1}\right|=\left|z_{2}\right|=\left|z_{3}\right|, \operatorname{Im}\left(z_{1} z_{2} z_{3}\right)=0, \operatorname{Re}\left(z_{1} z_{2} z_{3}\right) \geqslant 0\right\} .
$$

By [17, Ex. 3.5], $C$ is stable in the sense of Definition 2.8. Hence, SL 3-folds with conical singularities with cone $C$ have a very well-behaved deformation theory, by Theorem 2.10. We make our first conjecture.

Conjecture 5.5. The SL fibrations $F, F^{\prime}$ of Theorems 5.1, 5.3 are generic local models for codimension one singularities of SL fibrations $f: M \rightarrow B$ of almost Calabi-Yau 3-folds, using 'generic' as in Definition 3.6.

Here I am being deliberately vague on what I mean by a local model for an SL fibration. Roughly speaking, I want $f$ to have the same topological structure as $F$ or $F^{\prime}$ locally, for the fibres of $f$ to approximate those of $F$ or $F^{\prime}$ near the singular points, and for the singular fibres to have conical singularities with cone $C$, in the sense of Definition 2.9.

We can use the results of [17] to give a partial proof of Conjecture 5.5. Let $(M, J, \omega, \Omega)$ be an almost Calabi-Yau 3-fold, and $f: M \rightarrow B$ an SL fibration with generic fibre $T^{3}$, which is locally modelled on $F$ or $F^{\prime}$ near some singular point $x \in M$. Let $b=f(x)$ and $X=f^{-1}(b)$ be the fibre through $x$. Then $X$ is locally modelled on $C$ near $x$.

Suppose that $b$ is generic in the discriminant of $F$ or $F^{\prime}$, and that $X$ has only finitely many singularities $x=x_{1}, \ldots, x_{n}$, each modelled on $C$. Then [17, Th. 4.5] shows that $X$ has conical singularities at $x_{1}, \ldots, x_{n}$ with cone $C$, in the sense of Definition 2.9. The moduli space $\mathcal{M}_{X}$ of Theorem 2.10 locally coincides with the discriminant of $F$ or $F^{\prime}$. Hence it is smooth with $\operatorname{dim} \mathcal{M}_{X}=\operatorname{dim} \mathcal{I}_{X^{\prime}}=2$.

Now let $\tilde{\omega}$ be a Kähler form on $M$ close to $\omega$ and in the same Kähler class, as in Definition 3.6. As $C$ is stable, [14, Cor. 7.10] shows that for $\tilde{\omega}$ sufficiently close to $\omega$ there exists a deformation $\tilde{X}$ of $X$, which is an SL $m$-fold in $(M, J, \tilde{\omega}, \Omega)$ with conical singularities $\tilde{x}_{1}, \ldots, \tilde{x}_{n}$ and cone $C$, and lies in a smooth moduli space $\mathcal{M}_{\tilde{X}}$ with $\operatorname{dim} \mathcal{M}_{\tilde{X}}=2$.

We can then use the desingularization results of $[15,16]$, following $[17$, $\S 10]$, to show that $\tilde{X}$ must admit two families $\mathcal{F}_{\tilde{X}}^{ \pm}$of desingularizations, which are SL $T^{3}$ 's in $(M, J, \tilde{\omega}, \Omega)$, corresponding to the regions $a>0, a<0$ in $F$ or $F^{\prime}$. We expect that $\mathcal{M}_{\tilde{X}}, \mathcal{F}_{\tilde{X}}^{+}$and $\mathcal{F}_{\tilde{X}}^{-}$will locally form the fibres of 
an $S L$ fibration $\tilde{f}: M \rightarrow B$ of $(M, J, \tilde{\omega}, \Omega)$ close to $f$ and modelled on $F$ or $F^{\prime}$ near $x$, for $\tilde{\omega}$ sufficiently close to $\omega$.

Finally we discuss holomorphic discs with boundary in the fibres of $F, F^{\prime}$.

Lemma 5.6. Let $a>0$ and $c \in \mathbb{C}$. Then $\left\{\left(z_{1}, 0, c\right): z_{1} \in \mathbb{C},\left|z_{1}\right|^{2} \leqslant 2 a\right\}$ is a holomorphic disc in $\mathbb{C}^{3}$ with boundary in both $N_{a, c}$ and $N_{a, c}^{\prime}$, of area $2 \pi a$.

Similarly, let $a<0$ and $c \in \mathbb{C}$. Then $\left\{\left(0, z_{2}, c\right): z_{2} \in \mathbb{C},\left|z_{2}\right|^{2} \leqslant-2 a\right\}$ is a holomorphic disc in $\mathbb{C}^{3}$ with boundary in both $N_{a, c}$ and $N_{a, c}^{\prime}$, of area $-2 \pi a$.

The proof is trivial. Now if $D$ is a holomorphic disc in an almost CalabiYau 3-fold $(M, J, \omega, \Omega)$ with boundary in an SL 3-fold $L$ in $M$, then the area of $D$ is $\int_{D} \omega=[\omega] \cdot[D]$, where $[\omega] \in H^{2}(M, L ; \mathbb{R})$ is the relative de Rham cohomology class of $\omega$, and $[D] \in H_{2}(M, L ; \mathbb{Z})$ is the relative homology class of $D$. Thus the area of a holomorphic disc is essentially a topological invariant.

Holomorphic discs $D$ with boundary in SL 3-folds $L$ are typically stable objects which persist under small deformations of $L$. Also, the area of $D$ is always positive. Suppose we deform $L$ in $M$ so that the area $[\omega] \cdot[D]$ of $D$ shrinks to zero. Then $D$ shrinks down to a point, so that the boundary $\partial D$ in $L$, a circle, is collapsed to a point. So $L$ develops a singularity, a $T^{2}$-cone, by collapsing an $\mathcal{S}^{1}$ in $L$ to a point.

We can think of the singularities of the fibrations $F, F^{\prime}$ as occurring when the areas $2 \pi|a|$ of the holomorphic discs in Lemma 5.6 with boundary in the fibres of $F, F^{\prime}$ shrink to zero, at $a=0$. This should also be something which happens in SL fibrations of almost Calabi-Yau 3-folds, when holomorphic discs with boundaries in the fibres shrink to a point.

\section{A model for codimension 2 singular behaviour.}

Theorems 5.1 and 5.3 modelled the singular behaviour the author expects to occur in codimension one in generic SL fibrations. We shall now construct a model for the next most generic kind of singular behaviour, which occurs in codimension two in generic SL fibrations.

Unfortunately we cannot write the fibration down explicitly, so we will construct it using the analytic results of $\S 4$ and describe its properties. We begin by defining a family of solutions $\left(\hat{u}_{a, \alpha}, \hat{v}_{a, \alpha}\right)$ of $(10)$.

Definition 6.1. Let $D$ be the unit disc $\left\{(x, y) \in \mathbb{R}^{2}: x^{2}+y^{2} \leqslant 1\right\}$ in $\mathbb{R}^{2}$, with boundary $\mathcal{S}^{1}$, the unit circle. Define a coordinate $\theta: \mathbb{R} / 2 \pi \mathbb{Z} \rightarrow \mathcal{S}^{1}$ by $\theta \mapsto(\cos \theta, \sin \theta)$. Then $\cos (j \theta), \sin (j \theta) \in C^{\infty}\left(\mathcal{S}^{1}\right)$ for $j \geqslant 1$. 
For all $a, \alpha \in \mathbb{R}$ let $\hat{f}_{a, \alpha}$ be the unique solution of (17) in $D$ (with weak derivatives) given by Theorem 4.7, with this value of $a$ and boundary condition

$$
\left.\hat{f}_{a, \alpha}\right|_{\mathcal{S}^{1}}=\hat{\phi}_{\alpha}=\alpha \cos \theta-\cos (3 \theta) .
$$

Then $\hat{f}_{a, \alpha} \in C^{\infty}(D)$ if $a \neq 0$ and $\hat{f}_{0, \alpha} \in C^{1}(D)$. Define $\hat{u}_{a, \alpha}=\frac{\partial}{\partial y} \hat{f}_{a, \alpha}$ and $\hat{v}_{a, \alpha}=\frac{\partial}{\partial x} \hat{f}_{a, \alpha}$. Then $\left(\hat{u}_{a, \alpha}, \hat{v}_{a, \alpha}\right)$ is a solution of $(10)$ in $C^{\infty}(D)$ if $a \neq 0$, and a singular solution of $(9)$ in $C^{0}(D)$ if $a=0$, in the sense of Definition 4.3.

Theorem 6.2. These solutions $\left(\hat{u}_{a, \alpha}, \hat{v}_{a, \alpha}\right)$ have the following properties:

(i) $\hat{u}_{a, \alpha}(x,-y) \equiv-\hat{u}_{a, \alpha}(x, y)$ and $\hat{v}_{a, \alpha}(x,-y) \equiv \hat{v}_{a, \alpha}(x, y)$.

(ii) $\hat{u}_{a, \alpha}(-x, y) \equiv-\hat{u}_{a, \alpha}(x, y)$ and $\hat{v}_{a, \alpha}(-x, y) \equiv \hat{v}_{a, \alpha}(x, y)$.

(iii) $\hat{u}_{-a, \alpha}(x, y) \equiv \hat{u}_{a, \alpha}(x, y)$ and $\hat{v}_{-a, \alpha}(x, y) \equiv \hat{v}_{a, \alpha}(x, y)$.

(iv) There exists $C>0$ with $\left|\hat{u}_{a, \alpha}\right| \leqslant C$ and $\left|\hat{v}_{a, \alpha}-\alpha\right| \leqslant C$ on $D$ for all $a, \alpha$.

(v) If $a \in \mathbb{R},(b, c) \in D^{\circ}$ and $\alpha<\alpha^{\prime}$ then $\hat{v}_{a, \alpha}(b, c)<\hat{v}_{a, \alpha^{\prime}}(b, c)$.

(vi) For all $a, \alpha \in \mathbb{R}$ the function $x \mapsto \hat{v}_{a, \alpha}(x, 0)$ is strictly increasing for $x \in[-1,0]$, and strictly decreasing for $x \in[0,1]$.

(vii) For all $a, \alpha \in \mathbb{R}$ the function $y \mapsto \hat{v}_{a, \alpha}(0, y)$ is strictly decreasing for $y \in[-1,0]$, and strictly increasing for $y \in[0,1]$.

Proof. As $\hat{\phi}_{\alpha}$ has the symmetries $\hat{\phi}_{\alpha}(x,-y)=-\hat{\phi}_{\alpha}(-x, y)=\hat{\phi}_{\alpha}(x, y)$, uniqueness in Theorem 4.7 gives $\hat{f}_{a, \alpha}(x,-y)=-\hat{f}_{a, \alpha}(-x, y)=\hat{f}_{a, \alpha}(x, y)$, and parts (i), (ii) follow by taking partial derivatives. Part (iii) holds as (10) depends only on $a^{2}$ rather than $a$, and $\hat{\phi}_{\alpha}$ is independent of $a$.

An important part of the proof of Theorem 4.7 in $[9,10]$ was to derive $a$ priori estimates for $\|u\|_{C^{0}}$ and $\|v\|_{C^{0}}$ in terms of $\|\phi\|_{C^{2}}$. This was done by using functions of the form $\beta x+\gamma y+\delta$ as super- and subsolutions for $f$ at each point of $\partial S$, and so derive a bound for $|\partial f|$ on $\partial S$. As the maxima of $u, v$ occur on $\partial S$, this implies bounds for $|u|,|v|$ on $S$.

Now in our case this can be done uniformly in $\alpha$. That is, if $\beta x+\gamma y+\delta \leqslant$ $-\cos (3 \theta)$ on $\mathcal{S}^{1}$ then $(\alpha+\beta) x+\gamma y+\delta \leqslant \hat{\phi}_{\alpha}$ on $\mathcal{S}^{1}$ for all $\alpha$, and therefore $(\alpha+\beta) x+\gamma y+\delta \leqslant \hat{f}_{a, \alpha}$ on $D$ for all $a, \alpha$, and a similar statement for supersolutions. Following the proof of [9, Th. 3.9], we easily deduce part (iv).

Suppose $a \in \mathbb{R},(b, c) \in D^{\circ}, \alpha<\alpha^{\prime}$ and $\hat{v}_{a, \alpha}(b, c)=\hat{v}_{a, \alpha^{\prime}}(b, c)$. Define

$$
\begin{aligned}
& u_{1}(x, y)=\hat{u}_{a, \alpha}(x, y)+\hat{u}_{a, \alpha^{\prime}}(b, c)-\hat{u}_{a, \alpha}(b, c), \quad v_{1}(x, y)=\hat{v}_{a, \alpha}(x, y), \\
& u_{2}(x, y)=\hat{u}_{a, \alpha^{\prime}}(x, y) \quad \text { and } v_{2}(x, y)=\hat{v}_{a, \alpha^{\prime}}(x, y) \text {. }
\end{aligned}
$$


Then $\left(u_{j}, v_{j}\right)$ satisfy $(10)$ in $D$, and $\left(u_{1}, v_{1}\right)=\left(u_{2}, v_{2}\right)$ at $(b, c) \in D^{\circ}$.

Furthermore, $\left(u_{1}, v_{1}\right),\left(u_{2}, v_{2}\right)$ come from Theorem 4.7 with boundary data

$\phi_{1}=\alpha \cos \theta-\cos (3 \theta)+\left(\hat{u}_{a, \alpha^{\prime}}(b, c)-\hat{u}_{a, \alpha}(b, c)\right) \sin \theta$ and $\phi_{2}=\alpha^{\prime} \cos \theta-\cos (3 \theta)$.

Thus $\phi_{1}-\phi_{2}$ is a nontrivial linear combination of $\cos \theta, \sin \theta$, and has exactly 1 local maximum and 1 local minimum on $\mathcal{S}^{1}$. Applying Theorem 4.10 with $l=1$ shows that $\left(u_{1}, v_{1}\right)-\left(u_{2}, v_{2}\right)$ has no zeroes in $D^{\circ}$. But this contradicts $\left(u_{1}, v_{1}\right)=\left(u_{2}, v_{2}\right)$ at $(b, c)$.

So given $a \in \mathbb{R}$ and $(b, c) \in D^{\circ}$, whenever $\alpha<\alpha^{\prime}$ we have $\hat{v}_{a, \alpha}(b, c) \neq$ $\hat{v}_{a, \alpha^{\prime}}(b, c)$. Since $\hat{v}_{a, \alpha}(b, c)$ depends continuously on $\alpha, a, b, c$ it follows that $v_{a, \alpha}(b, c)$ is either a strictly increasing or a strictly decreasing function of $\alpha$, and which of the two is independent of $a, b, c$. Then (iv) shows it is increasing, proving (v).

For $\beta \in \mathbb{R}$, we can consider $(0, \beta)$ to be a solution of $(10)$ on $D$, coming from Theorem 4.7 with $f=\beta x$ and boundary data $\phi=\beta \cos \theta$. Now $\alpha \cos \theta-$ $\cos (3 \theta)-\beta \cos \theta$ has at most 3 local maxima and 3 local minima on $\mathcal{S}^{1}$ by [11, Prop. 10.2]. Hence, applying Theorem 4.10 with $l=3$ shows that $\left(\hat{u}_{a, \alpha}, \hat{v}_{a, \alpha}\right)-(0, \beta)$ has at most two zeroes in $D^{\circ}$, for any $\beta \in \mathbb{R}$.

We shall use this to show that

(a) Suppose $|x|,\left|x^{\prime}\right|<1$ and $\hat{v}_{a, \alpha}(x, 0)=\hat{v}_{a, \alpha}\left(x^{\prime}, 0\right)$. Then $x= \pm x^{\prime}$.

(b) Suppose $0<|x|,|y|<1$. Then $\hat{v}_{a, \alpha}(x, 0) \neq \hat{v}_{a, \alpha}(0, y)$.

Part (i) gives $\hat{u}_{a, \alpha}(x, 0) \equiv 0$. So if $|x|,\left|x^{\prime}\right|<1$ and $\hat{v}_{a, \alpha}(x, 0)=\hat{v}_{a, \alpha}\left(x^{\prime}, 0\right)=\beta$ then $\left(\hat{u}_{a, \alpha}, \hat{v}_{a, \alpha}\right)=(0, \beta)$ at $( \pm x, 0)$ and $\left( \pm x^{\prime}, 0\right)$, by part (ii). As there are at most two such points we must have $x= \pm x^{\prime}$, proving (a).

Similarly, (ii) gives $\hat{u}_{a, \alpha}(0, y) \equiv 0$. Thus if $0<|x|,|y|<1$ and $\hat{v}_{a, \alpha}(x, 0)=$ $\hat{v}_{a, \alpha}(0, y)=\beta$ then by (i), (ii) we see that $\left(\hat{u}_{a, \alpha}, \hat{v}_{a, \alpha}\right)=(0, \beta)$ at the four points $( \pm x, 0)$ and $(0, \pm y)$, a contradiction. This proves $(\mathrm{b})$.

Now from (a), (b) and the continuity of $\hat{v}_{a, \alpha}$ it is not difficult to see that either (vi), (vii) hold, or (vi), (vii) hold, but swapping 'increasing' and 'decreasing' throughout. But

$$
\int_{-1}^{1} \hat{v}_{a, \alpha}(x, 0) \mathrm{d} x=\hat{\phi}_{\alpha}(1,0)-\hat{\phi}_{\alpha}(-1,0)=2 \alpha-2,
$$

so the average of $\hat{v}_{a, \alpha}$ on the $x$-axis is $\alpha-1$, and

$$
\hat{v}_{a, \alpha}(0,1)=\frac{\partial \hat{f}_{a, \alpha}}{\partial x}(0,1)=-\frac{\mathrm{d} \hat{\phi}_{\alpha}}{\mathrm{d} \theta}(0,1)=\alpha+3 .
$$


Therefore $\hat{v}_{a, \alpha}$ is greater on the $y$-axis than on the $x$-axis, and so (vi) and (vii) hold, rather than their opposites.

Next we identify the singularities of $\left(\hat{u}_{0, \alpha}, \hat{v}_{0, \alpha}\right)$.

Proposition 6.3. There exist unique $\alpha_{0}<\alpha_{1}$ in $\mathbb{R}$ such that:

(i) If $\alpha \notin\left[\alpha_{0}, \alpha_{1}\right]$ then $\left(\hat{u}_{0, \alpha}, \hat{v}_{0, \alpha}\right)$ has no singularities in $D$.

(ii) $\left(\hat{u}_{0, \alpha_{0}}, \hat{v}_{0, \alpha_{0}}\right)$ has a singularity of multiplicity 2 and maximum type at $(0,0)$, and no other singularities.

(iii) If $\alpha \in\left(\alpha_{0}, \alpha_{1}\right)$ there exists $x \in(0,1)$ such that $\left(\hat{u}_{0, \alpha_{0}}, \hat{v}_{0, \alpha_{0}}\right)$ has a singularity of multiplicity 1 and increasing type at $(-x, 0)$, a singularity of multiplicity 1 and decreasing type at $(x, 0)$, and no other singularities.

(iv) $\left(\hat{u}_{0, \alpha_{1}}, \hat{v}_{0, \alpha_{1}}\right)$ is singular at $( \pm 1,0)$ on $\partial D$, and has no other singularities.

Proof. This follows quickly from Theorem 6.2 using the Intermediate Value Theorem, except for the multiplicities of the singular points. To find these we apply Theorem 4.16 to $\left(\hat{u}_{0, \alpha}, \hat{v}_{0, \alpha}\right)$. We have $\phi-\phi^{\prime}=2 \alpha \cos \theta-2 \cos (3 \theta)$, with at most 3 local maxima and 3 local minima on $\mathcal{S}^{1}$ by [11, Prop. 10.2]. So Theorem 4.16 shows that there are at most two singularities of $\left(\hat{u}_{0, \alpha}, \hat{v}_{0, \alpha}\right)$ in $D^{\circ}$, counted with multiplicity. Multiplicity 1 in (iii) follows at once, and multiplicity 2 in (ii) from Proposition 4.15 , as $(0,0)$ is of maximum type.

Next we use the results of $\S 4.5$ to construct the special Lagrangian fibration we want. We apply Example 4.11 with $S=D$ and $\phi=-\cos (3 \theta)$. Equivalently, we apply Definition 4.8 and Theorem 4.9 with $S=D$ and $\Phi(a, \alpha, \beta)=\alpha \cos \theta+\beta \sin \theta-\cos (3 \theta)$. Writing the definition and theorem out explicitly in our case gives:

Definition 6.4. For each $a, \alpha, \beta \in \mathbb{R}$, define $\hat{N}_{a, \alpha, \beta}$ in $\mathbb{C}^{3}$ by

$$
\begin{gathered}
\hat{N}_{a, \alpha, \beta}=\left\{\left(z_{1}, z_{2}, z_{3}\right) \in \mathbb{C}^{3}:\left|z_{1}\right|^{2}-\left|z_{2}\right|^{2}=2 a, x, y \in \mathbb{R}, x^{2}+y^{2}<1,\right. \\
\left.z_{1} z_{2}=\hat{v}_{a, \alpha}(x, y)+i y, \quad z_{3}=x+i \hat{u}_{a, \alpha}(x, y)+i \beta\right\} .
\end{gathered}
$$

Then $\hat{N}_{a, \alpha, \beta}$ is a noncompact SL 3 -fold without boundary in $\mathbb{C}^{3}$. 
Theorem 6.5. In the situation above, distinct $\hat{N}_{a, \alpha, \beta}$ are disjoint. Define

$$
V=\left\{\left(z_{1}, z_{2}, z_{3}\right) \in \mathbb{C}^{3}:\left(\operatorname{Re} z_{3}\right)^{2}+\left(\operatorname{Im} z_{1} z_{2}\right)^{2}<1\right\} .
$$

Then there exists a continuous, surjective $\hat{F}: V \rightarrow \mathbb{R}^{3}$ with $\hat{F}^{-1}(a, \alpha, \beta)=$ $\hat{N}_{a, \alpha, \beta}$ for all $(a, \alpha, \beta) \in \mathbb{R}^{3}$. Thus, $\hat{F}$ is a special Lagrangian fibration of $V$.

Proposition 6.3 gives the discriminant of $\hat{F}$.

Corollary 6.6. In the situation above, the discriminant of $\hat{F}$ is

$$
\hat{\Delta}=\left\{(0, \alpha, \beta): \alpha \in\left[\alpha_{0}, \alpha_{1}\right), \quad \beta \in \mathbb{R}\right\},
$$

and the set of singular points is $\left\{\left(0,0, z_{3}\right): z_{3} \in \mathbb{C},\left|\operatorname{Re} z_{3}\right|<1\right\}$.

\subsection{Discussion.}

We have constructed an SL fibration $\hat{F}$ in which the discriminant $\hat{\Delta}$ is a ribbon, a portion of a plane in $\mathbb{R}^{3}$. As in $\S 5, \hat{\Delta}$ is of real codimension one in the base $\mathbb{R}^{3}$. However, we are particularly interested in what happens at the boundary $\left\{\left(0, \alpha_{0}, \beta\right): \beta \in \mathbb{R}\right\}$ of $\hat{\Delta}$, which is of real codimension two in $\mathbb{R}^{3}$.

In the interior of $\hat{\Delta}$, each singular fibre $\hat{N}_{0, \alpha, \beta}$ with $\alpha \in\left(\alpha_{0}, \alpha_{1}\right)$ has two singular points, both of multiplicity 1 and one each of increasing and decreasing type. Locally these singularities are modelled on SL $T^{2}$-cones like $C$ in (29). As $\alpha \rightarrow \alpha_{0}$, these two singular points come together, until at $\alpha=\alpha_{0}$ they fuse to form a different kind of singularity, of multiplicity 2 . For $\alpha<\alpha_{0}$ there are no singularities. Thus the picture is that as $\alpha$ decreases through $\alpha_{0}$, the two singular points in $\hat{N}_{0, \alpha, \beta}$ come together and cancel out. As $\alpha$ increases through $\alpha_{1}$ the singular points cross the boundary of $V$.

Now consider the set of singular points $\left\{\left(0,0, z_{3}\right): z_{3} \in \mathbb{C},\left|\operatorname{Re} z_{3}\right|<1\right\}$. When $\operatorname{Re} z_{3} \in(-1,0)$, we see from part (iii) of Proposition 6.3 that the fibre of $\hat{F}$ passing through $\left(0,0, z_{3}\right)$ has a singularity of multiplicity 1 and increasing type, like those of $F$ in Theorem 5.1. When $\operatorname{Re} z_{3} \in(0,1)$, the fibre of $\hat{F}$ through $\left(0,0, z_{3}\right)$ has a singularity of multiplicity 1 and decreasing type, like those of $F^{\prime}$ in Theorem 5.3.

So the situation is that near $\left\{\left(0,0, z_{3}\right): z_{3} \in \mathbb{C}\right.$, Re $\left.z_{3} \in(-1,0)\right\}$, the fibration $\hat{F}$ is locally modelled on $F$ in Theorem 5.1, and near $\left\{\left(0,0, z_{3}\right)\right.$ : $\left.z_{3} \in \mathbb{C}, \operatorname{Re} z_{3} \in(0,1)\right\}$, the fibration $\hat{F}$ is locally modelled on $F^{\prime}$ in Theorem 5.3. On the line $\left\{\left(0,0, z_{3}\right): z_{3} \in \mathbb{C}, \operatorname{Re} z_{3}=0\right\}$, we have multiplicity 2 singularities, which mark the transition between the $F$ and $F^{\prime}$ local models.

I claim that $\hat{F}$ is a local model for generic SL fibrations. 
Conjecture 6.7. The $S L$ fibration $\hat{F}$ of Theorem 6.5 is a generic local model for one kind of codimension two singularities of $S L$ fibrations $f: M \rightarrow B$ of almost Calabi-Yau 3-folds, using 'generic' in the sense of Definition 3.6.

Again, I am not defining what I mean by a 'local model' here, but basically the fibrations should have the same topological structure and the same kinds of singularities. From above, for $\alpha \in\left(\alpha_{0}, \alpha_{1}\right)$ the singular fibres $\hat{N}_{0, \alpha, \beta}$ have two singular points modelled on the SL $T^{2}$-cone $C$ in (29). Thus it is an SL 3-fold with conical singularities, as in Definition 2.9 .

Therefore, as in $\S 5.1$, we can use the material of $\S 2.3$ to give a partial proof of the genericity of $\hat{F}$ around the fibres $\hat{N}_{0, \alpha, \beta}$ for $\alpha \in\left(\alpha_{0}, \alpha_{1}\right)$. However, the singularities of $\hat{N}_{0, \alpha_{0}, \beta}$ are not conical in the sense of Definition 2.9, so this approach will not help around $\alpha=\alpha_{0}$.

We shall discuss one feature of these fibrations, and why it is generic, in more detail. For $\alpha \in\left(\alpha_{0}, \alpha_{1}\right)$ the fibre $\hat{N}_{0, \alpha, \beta}$ has two singular points. $A$ priori this seems unlikely: distinct singular points ought to occur independently, and so for a fibre to have two codimension one singular points should be a codimension two phenomenon, not codimension one as in $\hat{F}$. However, this is not the case.

We can explain this in terms of holomorphic discs, as in $§ 5.1$. Let us identify the holomorphic discs in $\mathbb{C}^{3}$ with boundary in $\hat{N}_{a, \alpha, \beta}$.

Lemma 6.8. Let $a>0, \alpha, \beta \in \mathbb{R}$ and $x \in(0,1)$ with $\hat{v}_{a, \alpha}(x, 0)=0$. Then

(a) $D_{ \pm}=\left\{\left(z_{1}, 0, \pm x+i \hat{u}_{a, \alpha}(x, 0)+i \beta\right): z_{1} \in \mathbb{C},\left|z_{1}\right|^{2} \leqslant 2 a\right\}$ are two holomorphic discs with boundary in $\hat{N}_{a, \alpha, \beta}$ and area $2 \pi a$, and

(b) $D_{ \pm}^{\prime}=\left\{\left(0, z_{2}, \pm x+i \hat{u}_{a, \alpha}(x, 0)+i \beta\right): z_{2} \in \mathbb{C},\left|z_{2}\right|^{2} \leqslant 2 a\right\}$ are two holomorphic discs with boundary in $\hat{N}_{-a, \alpha, \beta}$ and area $2 \pi a$.

The proof is trivial. Suppose now that $a>0$ is small, $\alpha \in\left(\alpha_{0}, \alpha_{1}\right)$ and $\beta \in \mathbb{R}$. Then by Theorem 6.2 and Proposition 6.3 there exists $x \in(0,1)$ with $\hat{v}_{a, \alpha}(x, 0)=0$, and Lemma 6.8 gives holomorphic discs $D_{ \pm}$with boundary in $\hat{N}_{a, \alpha, \beta}$, and area $2 \pi a$. These discs are homologous in $H_{2}\left(\mathbb{C}^{3}, \hat{N}_{a, \alpha, \beta} ; \mathbb{R}\right)$. As a decreases to zero $D_{ \pm}$collapse to points, and $\hat{N}_{a, \alpha, \beta}$ develops two singular points.

Recall from $\S 5.1$ that if $D$ is a holomorphic disc in an almost Calabi-Yau 3 -fold $(M, J, \omega, \Omega)$ with boundary in an SL 3 -fold $L$ in $M$, then the area of $D$ is $[\omega] \cdot[D]$, where $[D] \in H_{2}(M, L ; \mathbb{Z})$ is the relative homology class of $D$. Therefore, if holomorphic discs $D_{1}, \ldots, D_{k}$ with boundary in $L$ have the same homology class in $H_{2}(M, L ; \mathbb{Z})$ then they have the same area. If we 
deform $L$ so that this area becomes zero, then $D_{1}, \ldots, D_{k}$ will simultaneously collapse to points, and $L$ will develop $k$ singular points. For some rigorous results on this when $k=2$, see $[17, \S 10.3]$.

This shows that distinct singular points of SL 3-folds may not be independent. Instead, if a singular point results from the collapse of a holomorphic disc, then singular points from the collapse of homologous holomorphic discs will always occur together. This is why it is permissible for the fibres of $\hat{F}$ to have two singular points in codimension one, and for this to be generic.

We can also use this to explain why the discriminant $\hat{\Delta}$ can have a boundary. For $\alpha \in\left(\alpha_{0}, \alpha_{1}\right)$ and $a$ small, there are two holomorphic discs $D_{ \pm}$with boundary in $N_{a, \alpha, \beta}$. These discs have opposite sign, so that the number of holomorphic discs counted with signs is zero. As $\alpha$ decreases with $a$ fixed, it reaches a value $\alpha^{\prime} \approx \alpha_{0}$ with $v_{a, \alpha^{\prime}}(0,0)=0$, and then $D_{ \pm}$ come together and cancel out. For $\alpha<\alpha^{\prime}$ there are no holomorphic discs with boundary in $N_{a, \alpha, \beta}$.

Thus, if we pass through the hypersurface $a=0$ when $\alpha \in\left(\alpha_{0}, \alpha_{1}\right)$, two holomorphic discs collapse to two singularities. But if we decrease $a$ past $\alpha_{0}$, the two holomorphic discs cancel, and then we can pass through $a=0$ without a singularity, as there are no holomorphic discs to collapse.

\section{How smooth SL fibrations become non-smooth.}

Next we shall extend the results of $\S 4$ from strictly convex domains in $\mathbb{R}^{2}$ to solutions on a strip in $\mathbb{R}^{2}$ which are periodic under a group of translations. We shall use this to model what happens near an $\mathcal{S}^{1}$ singularity of a singular fibre of a smooth SL fibration, and so describe how smooth SL fibrations become non-smooth under small deformations.

\subsection{A class of periodic U(1)-invariant SL 3-folds.}

Here is the situation we shall work with.

Definition 7.1. Let $R, P>0$, and define $S=\left\{(x, y) \in \mathbb{R}^{2}:|y| \leqslant R\right\}$. We shall study functions $u, v: S \rightarrow \mathbb{R}$ which satisfy (9) or (10), and the periodicity condition

$$
u(x+P, y) \equiv u(x, y) \quad \text { and } \quad v(x+P, y) \equiv v(x, y) \quad \text { for all }(x, y) \in S
$$


The main point is that although $S$ is noncompact, equation (34) implies that $u, v$ are invariant under the $\mathbb{Z}$-action

$$
(x, y) \stackrel{n}{\longmapsto}(x+n P, y) \quad \text { for } n \in \mathbb{Z},
$$

so we can treat $u, v$ as functions on the annulus $S / \mathbb{Z}$. Since $S / \mathbb{Z}$ is compact, analytic methods which rely on compactness will still apply, such as existence results for the Dirichlet problem.

Here are two lemmas on the consequences of the periodicity conditions (34). The second is an analogue of Proposition 4.2.

Lemma 7.2. Let $R, P$ and $S$ be as above, $a \neq 0$, and $u, v \in C^{1}(S)$ satisfy (10) and (34). Then there is a unique $\gamma \in \mathbb{R}$ with

$$
\int_{0}^{P} v(x, y) \mathrm{d} x=\gamma P \quad \text { for all } y \in[-R, R] .
$$

Proof. Since $u, v$ are continuously differentiable and satisfy (10), we have

$\frac{\mathrm{d}}{\mathrm{d} y} \int_{0}^{P} v(x, y) \mathrm{d} x=\int_{0}^{P} \frac{\partial v}{\partial y}(x, y) \mathrm{d} x=\int_{0}^{P} \frac{\partial u}{\partial x}(x, y) \mathrm{d} x=u(P, y)-u(0, y)=0$,

using (34). Hence $\int_{0}^{P} v(x, y) \mathrm{d} x$ is independent of $y$, and (36) holds for some unique $\gamma$.

Lemma 7.3. Let $R, P$ and $S$ be as above, and $v \in C^{2}(S)$ satisfy (11) for $a \neq 0, v(x+P, y) \equiv v(x, y)$ for all $(x, y) \in S$, and $\int_{0}^{P} v(x, R) \mathrm{d} x=$ $\int_{0}^{P} v(x,-R) \mathrm{d} x$. Then there exists a unique $u \in C^{2}(S)$ with $u(0,0)=0$ such that $u, v$ satisfy $(10)$ and $u(x+P, y) \equiv u(x, y)$ for all $(x, y) \in S$.

Proof. The proof of Proposition 4.2 shows that there exists $u \in C^{2}(S)$, unique up to $u \mapsto u+c$, such that $u, v$ satisfy (10). Requiring $u(0,0)=0$ fixes $c$. As $v$ is periodic under (35) we see from (10) that $\partial u$ is periodic, and hence $u$ satisfies $u(x+P, y) \equiv u(x, y)+\delta$ for some $\delta \in \mathbb{R}$. We must show $\delta=0$. As $\frac{\partial u}{\partial x}=\frac{\partial v}{\partial y}$, this follows from

$$
\begin{aligned}
0 & =\int_{0}^{P} v(x, R) \mathrm{d} x-\int_{0}^{P} v(x,-R) \mathrm{d} x=\int_{0}^{P} \int_{-R}^{R} \frac{\partial v}{\partial y}(x, y) \mathrm{d} y \mathrm{~d} x \\
& =\int_{-R}^{R} \int_{0}^{P} \frac{\partial u}{\partial x}(x, y) \mathrm{d} x \mathrm{~d} y=\int_{-R}^{R}(u(P, y)-u(0, y)) \mathrm{d} y=2 R \delta .
\end{aligned}
$$


In $\S 6$ our main tool for constructing SL fibrations was Theorem 4.7, the Dirichlet problem for solutions $f$ of $(17)$. We could take the same approach in this situation, but it turns out to be more elegant to solve the Dirichlet problem for solutions $v$ of (11) instead.

Now $[9$, Th. 8.8$]$ proves existence and uniqueness for the Dirichlet problem for $v$ in (11) on domains in $\mathbb{R}^{2}$ when $a \neq 0$. The proof does not assume the domain is convex, or use the fact that domains are contractible. In fact the proof applies to the compact annulus $S / \mathbb{Z}$ without change. This gives:

Theorem 7.4. Let $R, P$ and $S$ be as above, $a \neq 0, k \geqslant 0$, and $\alpha \in(0,1)$. Suppose $\phi^{ \pm} \in C^{k+2, \alpha}(\mathbb{R})$ with $\phi^{ \pm}(x+P) \equiv \phi^{ \pm}(x)$. Then there exists a unique $v \in C^{k+2, \alpha}(S)$ satisfying $(11), v(x+P, y) \equiv v(x, y)$ and $v(x, \pm R) \equiv$ $\phi^{ \pm}(x)$.

Before we extend this to the case $a=0$, we prove some a priori estimates for solutions $u, v$ in terms of the boundary data $\phi^{ \pm}$for $v$.

Theorem 7.5. Let $R, P$ and $S$ be as above, $a \neq 0$ and $u, v \in C^{2}(S)$ satisfy (10), (34), and $u(0,0)=0$. Define $\phi^{ \pm} \in C^{2}(\mathbb{R})$ by $v(x, \pm R)=\phi^{ \pm}(x)$. Then

$$
\|v\|_{C^{0}}=\max \left(\left\|\phi^{+}\right\|_{C^{0}},\left\|\phi^{-}\right\|_{C^{0}}\right) \text { and }\left\|\frac{\partial v}{\partial x}\right\|_{C^{0}}=\max \left(\left\|\frac{\partial \phi^{+}}{\partial x}\right\|_{C^{0}},\left\|\frac{\partial \phi^{-}}{\partial x}\right\|_{C^{0}}\right) \text {. }
$$

Suppose further that $A, B>0$ with $|a| \leqslant A$, and $\left\|\phi^{ \pm}\right\|_{C^{2}} \leqslant B$. Then there exist $C, D>0$ depending only on $R, P, A, B$ such that $\|u\|_{C^{0}} \leqslant C$, $\left\|\left.\partial u\right|_{\partial S}\right\|_{C^{0}} \leqslant D$ and $\left\|\left.\partial v\right|_{\partial S}\right\|_{C^{0}} \leqslant D$.

Proof. Regard $v$ and $\frac{\partial v}{\partial x}$ as functions on the compact annulus $S / \mathbb{Z}$, as they are invariant under the $\mathbb{Z}$-action (35). Now using the maximum principle for elliptic equations of a certain form, [9, Cor. 4.4] shows that the maximum and minimum of $v$ on a domain $T$ occur on $\partial T$, and [9, Prop. 8.12] that the maximum of $\left|\frac{\partial v}{\partial x}\right|$ occurs on $\partial T$. These proofs are also valid on the compact annulus $S / \mathbb{Z}$. But $v(x, \pm R)=\phi^{ \pm}(x)$ and $\frac{\partial v}{\partial x}(x, \pm R)=\frac{\partial}{\partial x} \phi^{ \pm}(x)$ on $\partial(S / \mathbb{Z})$. Maximizing and minimizing then gives (37).

Next we estimate $|\partial u|,|\partial v|$ on $\partial S$, following [10, Prop. 8.6]. Observe that if $(x, y) \in S$ with $|y| \geqslant \frac{1}{2} R$ then

$$
\frac{1}{4} R^{2} \leqslant v(x, y)^{2}+y^{2}+a^{2} \leqslant B^{2}+R^{2}+A^{2},
$$

since $|v| \leqslant \max \left(\left\|\phi^{+}\right\|_{C^{0}},\left\|\phi^{-}\right\|_{C^{0}}\right) \leqslant B$ by $(37)$ and $\left\|\phi^{ \pm}\right\|_{C^{2}} \leqslant B$. It follows that we can treat (11) as a quasilinear elliptic equation on $v$, which is uniformly elliptic on $|y| \geqslant \frac{1}{2} R$, with constants of ellipticity depending only on $A, B$ and $R$. 
Now Gilbarg and Trudinger [2, Th. 14.1, p. 337] show that if $v \in C^{2}(T)$ satisfies a quasilinear equation $Q v=0$ of the form (7) on a domain $T$ and $\left.v\right|_{\partial T}=\phi \in C^{2}(\partial T)$, then $\left\|\left.\partial v\right|_{\partial T}\right\|_{C^{0}} \leqslant K$ for some $K>0$ depending only on $T$, upper bounds for $\|v\|_{C^{0}}$ and $\|\phi\|_{C^{2}}$, and certain constants to do with $Q$, which ensure that $Q$ is uniformly elliptic and $b$ not too large.

As this is a local result, it is enough for the conditions to hold within distance $\frac{1}{2} R$ of $\partial T$. So there exists $K>0$ depending only on $A, B$ and $R$ such that $\left\|\left.\partial v\right|_{\partial S}\right\|_{C^{0}} \leqslant K$. But $\partial v$ determines $\partial u$ by (10), and we easily deduce a bound for $\left\|\left.\partial u\right|_{\partial S}\right\|_{C^{0}}$. Thus there exists $D>0$ depending only on $A, B$ and $R$ such that $\left\|\left.\partial u\right|_{\partial S}\right\|_{C^{0}} \leqslant D$ and $\left\|\left.\partial v\right|_{\partial S}\right\|_{C^{0}} \leqslant D$.

Finally we estimate $\|u\|_{C^{0}}$. Since $u(x, R)$ is periodic in $x$ with period $P$ and $\left|\frac{\partial u}{\partial x}(x, R)\right| \leqslant D$, we see that the variation of $x \mapsto u(x, R)$ is at most $\frac{1}{2} P D$. Similarly, the variation of $x \mapsto u(x,-R)$ is at most $\frac{1}{2} P D$. Therefore $u$ varies only a bounded amount from its average value on $y=R$, and the same on $y=-R$. We need to bound the difference between these average values. To do this we use the method of $[10, \S 8.4]$ to bound $\left\|\frac{\partial u}{\partial y}\right\|_{L^{1}}$ on $S / \mathbb{Z}$.

Define $J(a, v)=-\int_{0}^{v}\left(w^{2}+a^{2}\right)^{-1 / 4} \mathrm{~d} w$. Then [10, Prop. 8.9] shows that

$$
\begin{aligned}
& \int_{0}^{P} \int_{-R}^{R}\left(v^{2}+a^{2}\right)^{-1 / 4}\left[\frac{1}{2}\left(v^{2}+y^{2}+a^{2}\right)^{-1 / 2}\left(\frac{\partial v}{\partial x}\right)^{2}+\left(\frac{\partial v}{\partial y}\right)^{2}\right] \mathrm{d} y \mathrm{~d} x= \\
& \int_{0}^{P} J(a, v(x, R)) \frac{\partial u}{\partial x}(x, R) \mathrm{d} x-\int_{0}^{P} J(a, v(x,-R)) \frac{\partial u}{\partial x}(x,-R) \mathrm{d} x .
\end{aligned}
$$

The proof is that $\int_{S / \mathbb{Z}} \mathrm{d}(J(a, v) \mathrm{d} u)=\int_{\partial(S / \mathbb{Z})} J(a, v) \mathrm{d} u$ by Stokes' Theorem, and using (10) to rewrite the l.h.s. gives (38). Therefore

$$
\begin{aligned}
& \left(\int_{0}^{P} \int_{-R}^{R}\left|\frac{\partial u}{\partial y}\right| \mathrm{d} y \mathrm{~d} x\right)^{2}\left(\int_{0}^{P} \int_{-R}^{R}\left(v^{2}+y^{2}+a^{2}\right)^{-1 / 4} \mathrm{~d} y \mathrm{~d} x\right)^{-1} \\
\leqslant & \int_{0}^{P} \int_{-R}^{R}\left(v^{2}+y^{2}+a^{2}\right)^{1 / 4}\left(\frac{\partial u}{\partial y}\right)^{2} \mathrm{~d} y \mathrm{~d} x \\
\leqslant & \frac{1}{2} \int_{0}^{P} J(a, v(x, R)) \frac{\partial u}{\partial x}(x, R) \mathrm{d} x-\frac{1}{2} \int_{0}^{P} J(a, v(x,-R)) \frac{\partial u}{\partial x}(x,-R) \mathrm{d} x,
\end{aligned}
$$

where the second line follows from Hölder's inequality, and the third from (38) and the inequality $\left(v^{2}+y^{2}+a^{2}\right)^{1 / 4}\left|\frac{\partial u}{\partial y}\right|^{2} \leqslant \frac{1}{4}\left(v^{2}+a^{2}\right)^{-1 / 4}\left(v^{2}+y^{2}+\right.$ $\left.a^{2}\right)^{-1 / 2}\left|\frac{\partial v}{\partial x}\right|^{2}$, which follows from (10).

Using $|a| \leqslant A,|v| \leqslant B$ and the methods of [10, Prop. 8.10] we may derive a priori estimates depending only on $A, B, R$ and $P$ for the third line of (39) and the second integral on the first line. This gives an upper bound, $E$ say, 
for $\int_{0}^{P} \int_{-R}^{R}\left|\frac{\partial u}{\partial y}\right| \mathrm{d} y \mathrm{~d} x$. But

$$
\left|\int_{0}^{P}(u(x, R)-u(x,-R)) \mathrm{d} x\right|=\left|\int_{0}^{P} \int_{-R}^{R} \frac{\partial u}{\partial y} \mathrm{~d} y \mathrm{~d} x\right| \leqslant \int_{0}^{P} \int_{-R}^{R}\left|\frac{\partial u}{\partial y}\right| \mathrm{d} y \mathrm{~d} x \leqslant E .
$$

Therefore the difference in the average values of $u$ on $y= \pm R$ is at most $E / P$. But the variation of $u$ on $y=R$ and on $y=-R$ is at most $\frac{1}{2} P D$, from above. It follows that the variation of $u$ on both lines $y= \pm R$ together is at most $C=E / P+P D$, which depends only on $A, B, R$ and $P$.

Now $u$ satisfies a maximum principle by $[9$, Cor. 4.4], and so the maximum and minimum of $u$ on the compact annulus $S / \mathbb{Z}$ occur on $\partial(S / \mathbb{Z})$. Hence the difference between the maximum and minimum of $u$ is the variation of $u$ on both lines $y= \pm R$ together, and is at most $C$. But $u(0,0)=0$, so the maximum is nonnegative, and the minimum nonpositive. Therefore the maximum is at most $C$ and the minimum at least $-C$, and $\|u\|_{C^{0}} \leqslant C$, completing the proof.

Our next result extends [10, Th. $8.17 \& 8.18]$.

Theorem 7.6. Let $R, P$ and $S$ be as above, $a \in \mathbb{R}, k \geqslant 0$, and $\alpha \in(0,1)$. Suppose $\phi^{+}, \phi^{-} \in C^{k+2, \alpha}(\mathbb{R})$ with $\phi^{ \pm}(x+P) \equiv \phi^{ \pm}(x)$ and $\int_{0}^{P} \phi^{+}(x) \mathrm{d} x=$ $\int_{0}^{P} \phi^{-}(x) \mathrm{d} x$. Then if $a \neq 0$ there exist unique $u, v \in C^{k+2, \alpha}(S)$ satisfying (10), (34), $u(0,0)=0$ and $v(x, \pm R) \equiv \phi^{ \pm}(x)$. If $a=0$ there exist unique $u, v \in C^{0}(S)$ which are a singular solution of (9) in the sense of Definition 4.3 , and satisfy $(34), u(0,0)=0$ and $v(x, \pm R) \equiv \phi^{ \pm}(x)$. Furthermore $u, v$ depend continuously in $C^{0}(S)$ on $\phi^{+}, \phi^{-}$in $C^{k+2, \alpha}(\mathbb{R})$ and a in $\mathbb{R}$.

Proof. When $a \neq 0$, existence and uniqueness of $v$ comes from Theorem 7.4, and of $u$ from Lemma 7.3. To extend this to the case $a=0$ by taking the limit $a \rightarrow 0_{+}$we follow [10, $\left.\S 8\right]$, using the a priori estimates of Theorem 7.5. There are few significant changes, and the problems caused by singular points on the boundary in [10] are absent in this case, as there are no points $(x, 0)$ on $\partial S$. The final part is proved as in [10, Th. 8.18].

Applying [9, Prop. 8.7] and [11, Th. 6.16] on the annulus $S / \mathbb{Z}$ proves:

Theorem 7.7. Let $R, P$ and $S$ be as above, $a \in \mathbb{R}, k \geqslant 0$ and $\alpha \in(0,1)$, and suppose $\phi_{i}^{ \pm} \in C^{k+2, \alpha}(\mathbb{R})$ for $i=1,2$ satisfy $\phi_{i}^{ \pm}(x+P)=\phi_{i}^{ \pm}(x)$ and $\phi_{1}^{ \pm}(x)<\phi_{2}^{ \pm}(x)$ for all $x \in \mathbb{R}$, and $\int_{0}^{P} \phi_{i}^{+}(x) \mathrm{d} x=\int_{0}^{P} \phi_{i}^{-}(x) \mathrm{d} x$. Let $\left(u_{i}, v_{i}\right)$ 
be the (singular) solution of (10) produced in Theorem 7.6 from $\phi_{i}^{ \pm}$for $i=$ 1,2. Then $v_{1}<v_{2}$ on $S$.

\subsection{Applications to SL fibrations.}

We can now prove analogues of Definition 4.8 and Theorem 4.9.

Definition 7.8. Let $R, P$ and $S$ be as above and $\alpha \in(0,1)$. Suppose $\phi_{a, b}^{+}, \phi_{a, b}^{-} \in C^{3, \alpha}(\mathbb{R})$ are given for all $a, b \in \mathbb{R}$ and satisfy

(i) $\phi_{a, b}^{ \pm}$depend continuously in $C^{3, \alpha}(\mathbb{R})$ on $a, b \in \mathbb{R}$,

(ii) $\phi_{a, b}^{ \pm}(x+P)=\phi_{a, b}^{ \pm}(x)$ for all $a, b, x \in \mathbb{R}$,

(iii) $\int_{0}^{P} \phi_{a, b}^{+}(x) \mathrm{d} x=\int_{0}^{P} \phi_{a, b}^{-}(x) \mathrm{d} x$ for all $a, b \in \mathbb{R}$,

(iv) If $b<b^{\prime}$ then $\phi_{a, b}^{ \pm}(x)<\phi_{a, b^{\prime}}^{ \pm}(x)$ for all $a, x \in \mathbb{R}$.

(v) For all $a, x \in \mathbb{R}$ we have $\phi_{a, b}^{+}(x), \phi_{a, b}^{-}(x) \rightarrow \pm \infty$ as $b \rightarrow \pm \infty$.

For all $a, b \in \mathbb{R}$, let $\left(u_{a, b}, v_{a, b}\right)$ be the (singular) solution of (10) produced in Theorem 7.6 from $\phi_{a, b}^{ \pm}$. Let $\mathbb{Z}$ act on $\mathbb{C}^{3}$ by

$$
\left(z_{1}, z_{2}, z_{3}\right) \stackrel{n}{\longmapsto}\left(z_{1}, z_{2}, z_{3}+n P\right) \quad \text { for } n \in \mathbb{Z},
$$

corresponding to (35). For all $a, b, c \in \mathbb{R}$ define $N_{a, b, c}$ in $\mathbb{C}^{3} / \mathbb{Z}$ by

$$
\begin{gathered}
N_{a, b, c}=\left\{\left(z_{1}, z_{2}, z_{3}\right) \in \mathbb{C}^{3}:\left|z_{1}\right|^{2}-\left|z_{2}\right|^{2}=2 a, \quad x \in \mathbb{R}, y \in(-R, R),\right. \\
\left.z_{1} z_{2}=v_{a, b}(x, y)+i y, \quad z_{3}=x+i u_{a, b}(x, y)+i c\right\} / \mathbb{Z} .
\end{gathered}
$$

Then $N_{a, b, c}$ is a noncompact SL 3 -fold without boundary in $\mathbb{C}^{3} / \mathbb{Z}$, which is nonsingular if $a \neq 0$, by Proposition 4.1.

Theorem 7.9. In the situation above, distinct $N_{a, b, c}$ are disjoint. Define

$$
V=\left\{\left(z_{1}, z_{2}, z_{3}\right) \in \mathbb{C}^{3}:\left|\operatorname{Im} z_{1} z_{2}\right|<R\right\} / \mathbb{Z} .
$$

Then there exists a continuous, surjective $F: V \rightarrow \mathbb{R}^{3}$ with $F^{-1}(a, b, c)=$ $N_{a, b, c}$ for all $(a, b, c) \in \mathbb{R}^{3}$. Thus, $F$ is a special Lagrangian fibration of $V$.

Proof. Clearly we have $N_{a, b, c} \subset V$ for each $(a, b, c) \in \mathbb{R}^{3}$, where $N_{a, b, c}$ and $V$ are as in (41) and (42). Let $\left(z_{1}, z_{2}, z_{3}\right) \mathbb{Z} \in V$. We shall show that there exists a unique $(a, b, c) \in \mathbb{R}^{3}$ such that $\left(z_{1}, z_{2}, z_{3}\right) \mathbb{Z} \in N_{a, b, c}$. Let $x=\operatorname{Re} z_{3}$, 
$y=\operatorname{Im} z_{1} z_{2}$ and $2 a=\left|z_{1}\right|^{2}-\left|z_{2}\right|^{2}$. Consider the function $b \mapsto v_{a, b}(x, y)$. By part (i) of Definition 7.8 and the last part of Theorem 7.6 this is a continuous function.

If $b<b^{\prime}$ then part (iv) of Definition 7.8 gives $\phi_{a, b}^{ \pm}<\phi_{a, b^{\prime}}^{ \pm}$, and so Theorem 7.7 gives $v_{a, b}<v_{a, b^{\prime}}$ on $S$. Thus $b \mapsto v_{a, b}(x, y)$ is strictly increasing. And as the maximum and minimum of $v_{a, b}$ on $S / \mathbb{Z}$ is achieved on $\partial(S / \mathbb{Z})$ and so is a value of $\phi_{a, b}^{ \pm}$, part (v) of Definition 7.8 implies that $v_{a, b}(x, y) \rightarrow \pm \infty$ as $b \rightarrow \pm \infty$.

Hence by the Intermediate Value Theorem there exists a unique $b \in \mathbb{R}$ such that $v_{a, b}(x, y)=\operatorname{Re} z_{1} z_{2}$, and then $c$ is given by $c=\operatorname{Im} z_{3}-u_{a, b}(x, y)$. It is easy to see from (41) that $\left(z_{1}, z_{2}, z_{3}\right) \mathbb{Z} \in N_{a, b, c}$, and that this is the only $(a, b, c) \in \mathbb{R}^{3}$ for which this holds. Therefore distinct $N_{a, b, c}$ are disjoint, and $V=\bigcup_{(a, b, c) \in \mathbb{R}^{3}} N_{a, b, c}$. The remainder of the proof follows Theorem 4.9.

Here is a simple, explicit example.

Example 7.10. In the situation above, define $\phi_{a, b}^{+}(x)=\phi_{a, b}^{-}(x)=b$ for all $a, b, x \in \mathbb{R}$. Then parts (i)-(v) of Definition 7.8 hold. It is easy to verify that $v_{a, b}(x, y) \equiv b$ and $u_{a, b}(x, y) \equiv 0$. Hence

$$
\begin{gathered}
N_{a, b, c}=\left\{\left(z_{1}, z_{2}, z_{3}\right) \in \mathbb{C}^{3}:\left|z_{1}\right|^{2}-\left|z_{2}\right|^{2}=2 a, \quad \operatorname{Re} z_{1} z_{2}=b, \quad \operatorname{Im} z_{3}=c,\right. \\
\left.\operatorname{Im} z_{1} z_{2} \in(-R, R)\right\} / \mathbb{Z} .
\end{gathered}
$$

It readily follows that the special Lagrangian fibration $F: V \rightarrow \mathbb{C}^{3}$ of Theorem 7.9 is given by

$$
F\left(\left(z_{1}, z_{2}, z_{3}\right) \mathbb{Z}\right)=\left(\frac{1}{2}\left|z_{1}\right|^{2}-\frac{1}{2}\left|z_{2}\right|^{2}, \operatorname{Re} z_{1} z_{2}, \operatorname{Im} z_{3}\right) .
$$

Note that $F$ is a smooth SL fibration.

It is easy to show that $N_{a, b, c}$ is singular if and only if $a=b=0$, so that the discriminant of $F$ is $\Delta=\{(0,0, c): c \in \mathbb{R}\}$. This is of codimension two in $\mathbb{R}^{3}$, as in Proposition 3.5. The singular set of $N_{0,0, c}$ is $\{(0,0, x+i c): x \in \mathbb{R}\} / \mathbb{Z}$, which is a circle $\mathcal{S}^{1}$ in $\mathbb{C}^{3} / \mathbb{Z}$.

\subsection{A 1-parameter family of SL fibrations.}

We now construct a 1-parameter family of SL fibrations $F^{t}$ for $t \in[0,1]$.

Example 7.11. Fix $P=2 \pi$, let $t \in[0,1]$, and define $\phi_{a, b}^{+}(x)=\phi_{a, b}^{-}(x)=$ $b+t \cos x$ for all $a, b, x \in \mathbb{R}$. Then parts (i)-(v) of Definition 7.8 hold. Let $F^{t}$ be the SL fibration constructed in Theorem 7.9 using this data. This gives 
a 1-parameter family of SL fibrations $F^{t}: V \rightarrow \mathbb{C}^{3}$ for $t \in[0,1]$, where $F^{0}$ coincides with the smooth SL fibration of (43). Use the notation $u_{a, b}^{t}, v_{a, b}^{t}$ and $N_{a, b, c}^{t}$ in the obvious way. By the last part of Theorem 7.6 the $N_{a, b, c}^{t}$ depend continuously on $t$, and thus the $F^{t}$ depend continuously on $t$.

From above, the singular fibres of $F^{0}$ are $N_{0,0, c}^{0}$, and each is singular along a circle $\mathcal{S}^{1}$. However, for $t>0$ things are different. By Theorem 4.13, a necessary condition for $N_{0, b, c}^{t}$ to have non-isolated singularities is that $v_{0, b}^{t}(x,-y) \equiv-v_{0, b}^{t}(x, y)$. But putting $y=R$ then gives $\phi_{0, b}^{-}(x) \equiv-\phi_{0, b}^{+}(x)$, which is false. Thus when $t>0$, each $N_{0, b, c}^{t}$ has isolated singularities, and only finitely many of them by compactness.

This will be our local model of how to deform a smooth SL fibration to a non-smooth SL fibration. Here are some facts we will need.

Proposition 7.12. In Example 7.11, for all $t \in(0,1]$ and $a, b \in \mathbb{R}$ we have

(a) $x \mapsto v_{a, b}^{t}(x, y)$ has period $2 \pi$ and is strictly decreasing on $[0, \pi]$ and strictly increasing on $[\pi, 2 \pi]$, for all $y \in[-R, R]$.

(b) $u_{a, b}^{t}(x, y)>0$ on $(0, \pi) \times(0, R]$ and $(\pi, 2 \pi) \times[-R, 0)$, and $u_{a, b}^{t}(x, y)<0$ on $(0, \pi) \times[-R, 0)$ and $(\pi, 2 \pi) \times(0, R]$.

Proof. By symmetries of the $\phi_{a, b}^{ \pm}$the $v_{a, b}^{t}$ satisfy $v_{a, b}^{t}(-x, y) \equiv v_{a, b}^{t}(x, y)$ and $v_{a, b}^{t}(2 \pi-x, y) \equiv v_{a, b}^{t}(x, y)$. Hence $\frac{\partial}{\partial x} v_{a, b}^{t}(0, y) \equiv \frac{\partial}{\partial x} v_{a, b}^{t}(\pi, y) \equiv 0$ for $a \neq 0$. Also $\frac{\partial}{\partial x} v_{a, b}^{t}(x, \pm R) \equiv-t \sin x$. Thus $\frac{\partial}{\partial x} v_{a, b}^{t}(x, \pm R)<0$ for $x \in(0, \pi)$ and $t>0$.

Now by $[9, \S 8.4], \frac{\partial}{\partial x} v_{a, b}^{t}$ satisfies a kind of maximum principle. Applying this on the rectangle $[0, \pi] \times[-R, R]$ and using the fact that $\frac{\partial}{\partial x} v_{a, b}^{t}$ is zero on two sides of the rectangle and negative on the other two, we find that $\frac{\partial}{\partial x} v_{a, b}^{t}<0$ on $(0, \pi) \times[-R, R]$ when $t>0$ and $a \neq 0$. Similarly, $\frac{\partial}{\partial x} v_{a, b}^{t}>0$ on $(\pi, 2 \pi) \times[-R, R]$ when $t>0$ and $a \neq 0$. This implies part (a) when $a \neq 0$.

Also, by (10) we see that $\frac{\partial}{\partial y} u_{a, b}^{t}>0$ on $(0, \pi) \times[-R, R]$ and $\frac{\partial}{\partial y} u_{a, b}^{t}<0$ on $(\pi, 2 \pi) \times[-R, R]$. By symmetries of the $\phi_{a, b}^{ \pm}$the $u_{a, b}^{t}$ satisfy $u_{a, b}^{t}(x,-y) \equiv$ $-u_{a, b}^{t}(x, y)$, and so $u_{a, b}^{t}(x, 0) \equiv 0$. Part (b) for $a \neq 0$ then follows by integration on the line segment $\{x\} \times[0, y]$.

Taking the limit $a \rightarrow 0$ in (a) shows that $x \mapsto v_{0, b}^{t}(x, y)$ is decreasing on $[0, \pi]$ and increasing on $[\pi, 2 \pi]$. If it were not strictly decreasing or increasing then it would be constant on some subinterval $[\alpha, \beta]$ of $[0,2 \pi]$ with $\alpha<\beta$. 
Then $\left(u_{0, b}^{t}, v_{0, b}^{t}\right)$ is constant on $[\alpha, \beta] \times\{0\}$, and [11, Th. 7.8] implies that it is constant on $S$, a contradiction. This completes part (a).

Similarly, taking the limit $a \rightarrow 0$ in (b) shows that $u_{0, b}^{t}(x, y) \geqslant 0$ on $(0, \pi) \times(0, R]$ and $(\pi, 2 \pi) \times[-R, 0)$ and $u_{0, b}^{t}(x, y) \leqslant 0$ on $(0, \pi) \times[-R, 0)$ and $(\pi, 2 \pi) \times(0, R]$. But if $u_{0, b}^{t}(x, y)=0$ at any interior point of these regions, then we can derive a contradiction using the strong maximum principle $[2$, Th. 3.5]. This completes part (b).

We can now prove an analogue of Proposition 6.3 for Example 7.11.

Proposition 7.13. In Example 7.11 there are continuous $\alpha, \beta:[0,1] \rightarrow \mathbb{R}$ with

$$
F^{t}((0,0,0) \mathbb{Z})=(0, \alpha(t), 0) \quad \text { and } \quad F^{t}((0,0, \pi) \mathbb{Z})=(0, \beta(t), 0),
$$

and $\alpha(0)=\beta(0)=0$. For all $t \in(0,1]$ these have the properties that

(i) If $b \notin[\alpha(t), \beta(t)]$ then $\left(u_{0, b}^{t}, v_{0, b}^{t}\right)$ has no singularities in $S$.

(ii) $\left(u_{0, \alpha(t)}^{t}, v_{0, \alpha(t)}^{t}\right)$ has singularities of multiplicity 2 and maximum type at $(2 n \pi, 0)$ for $n \in \mathbb{Z}$, and no other singularities.

(iii) $\left(u_{0, \beta(t)}^{t}, v_{0, \beta(t)}^{t}\right)$ has singularities of multiplicity 2 and minimum type at $(\pi+2 n \pi, 0)$ for $n \in \mathbb{Z}$, and no other singularities.

(iv) If $b \in(\alpha(t), \beta(t))$ there exists $x \in(0, \pi)$ such that $\left(u_{0, b}^{t}, v_{0, b}^{t}\right)$ has singularities of multiplicity 1 and increasing type at $(2 n \pi-x, 0)$ for $n \in \mathbb{Z}$, singularities of multiplicity 1 and decreasing type at $(2 n \pi+x, 0)$ for $n \in \mathbb{Z}$, and no other singularities.

Proof. Let $t \in[0,1]$. As in the proof of Theorem 7.9, the function $b \mapsto$ $v_{0, b}^{t}(0,0)$ is continuous, strictly increasing, and tends to $\pm \infty$ as $b \rightarrow \pm \infty$. Thus by the Intermediate Value Theorem there exists a unique value $\alpha(t)$ such that $v_{0, \alpha(t)}^{t}(0,0)=0$. Since $u_{0, \alpha(t)}^{t}(0,0)=0$ by definition, this means that $(0,0,0) \mathbb{Z} \in N_{0, \alpha(t), 0}^{t}$ by $(41)$, and so $F^{t}((0,0,0) \mathbb{Z})=(0, \alpha(t), 0)$ by definition of $F^{t}$.

Hence $\alpha:[0,1] \rightarrow \mathbb{R}$ exists, and is continuous as $F^{t}$ depends continuously on $t$. Similarly, considering the function $b \mapsto v_{0, b}^{t}(\pi, 0)$ we find a unique value $\beta(t)$ such that $v_{0, \beta(t)}^{t}(\pi, 0)=0$, and $\beta:[0,1] \rightarrow \mathbb{R}$ exists and is continuous. Also $F^{0}$ is given in (43), so $\alpha(0)=\beta(0)=0$ follows from (44). 
Let $t \in(0,1]$, and consider the function $x \mapsto v_{0, b}^{t}(x, 0)$. By part (a) of Proposition 7.12 this has period $2 \pi$ with a maximum at 0 and a minimum at $\pi$. But $b \mapsto v_{0, b}^{t}(0,0)$ is strictly increasing and zero when $b=\alpha(t)$. Hence the maximum of $x \mapsto v_{0, b}^{t}(x, 0)$ is negative when $b<\alpha(t)$, zero when $b=\alpha(t)$ and positive when $b>\alpha(t)$. In particular, if $b<\alpha(t)$ then $v_{0, b}^{t}(x, 0)<0$ for all $x$, so that $\left(u_{0, b}^{t}, v_{0, b}^{t}\right)$ has no singularities. This proves half of part (i).

By similar arguments using part (a) of Proposition 7.12 and the Intermediate Value Theorem, we easily find that the singularities of $\left(u_{0, b}^{t}, v_{0, b}^{t}\right)$, which are the zeroes of $x \mapsto v_{0, b}^{t}(x, 0)$, are as given in parts (i)-(iv), and the type of each singularity also follows from part (a) of Proposition 7.12. Finally, to identify the multiplicity of each singularity $(x, 0)$ we can use part (b) of Proposition 7.12, as knowing the sign of $u_{0, b}^{t}$ constrains the winding number of $\left(u_{0, b}^{t}, v_{0, b}^{t}\right)$ about 0 along $\gamma_{\epsilon}(x, 0)$ in Definition 4.12.

This gives the discriminant of $F^{t}$.

Corollary 7.14. In Example 7.11, the discriminant of $F^{t}$ is

$$
\Delta^{t}=\{(0, b, c): b \in[\alpha(t), \beta(t)], \quad c \in \mathbb{R}\} \subset \mathbb{R}^{3},
$$

and the set of singular points is $\left\{\left(0,0, z_{3}\right): z_{3} \in \mathbb{C}\right\} / \mathbb{Z}$. For $t \in(0,1]$, if $b=\alpha(t)$ or $b=\beta(t)$ then $N_{0, b, c}$ has one singular point, and if $\alpha(t)<b<\beta(t)$ then $N_{0, b, c}$ has two singular points.

Note that the set of singular points of $F^{t}$ in $\mathbb{C}^{3}$ is independent of $t$.

\subsection{Discussion.}

Example 7.11 constructs a continuous 1-parameter family of SL fibrations $F^{t}: V \rightarrow \mathbb{R}^{3}$ for $t \in[0,1]$, where $F^{0}$ is the smooth SL fibration given explicitly in (43), but $F^{t}$ is not smooth for $t \in(0,1]$. Thus, this provides a local model for how to continuously deform a smooth SL fibration to a non-smooth SL fibration.

We have now seen several examples of non-smooth SL fibrations, and a local mechanism for deforming smooth SL fibrations to non-smooth ones. This justifies the following:

Conjecture 7.15. Generic SL fibrations $f: M \rightarrow B$ of almost Calabi-Yau 3-folds including fibres with singularities are never smooth, but only piecewise smooth. Here we use 'generic' in the sense of Definition 3.6. 
Suppose we have an almost Calabi-Yau 3-fold $M$ with a smooth SL fibration $f: M \rightarrow B$. What happens to the fibration if we deform $M$ to a nearby generic almost Calabi-Yau 3-fold $\tilde{M}$ ? I believe that the SL fibration will still exist, at least on most of $\tilde{M}$, but that it will be only piecewise smooth.

For a smooth SL fibration the discriminant $\Delta$ is of codimension two, and is expected to be a graph. In Example 7.11, when $t=0$ and $F^{0}$ is smooth, the discriminant $\Delta^{0}$ is a line in $\mathbb{R}^{3}$, of codimension two, but as $t$ increases $\Delta^{t}$ thickens out continuously into a ribbon in $\mathbb{R}^{3}$, of codimension one. In the same way, in a small generic deformation $\tilde{M}$ of $M$, I conjecture that the edges of the graph $\Delta$ in $B$ thicken out into 2-dimensional ribbons in $\tilde{\Delta} \subset B$. We will discuss what happens near the vertices of $\Delta$ in $\S 8$.

We can also relate the behaviour of $F^{t}$ to the local models of $\S 5$ and $\S 6$ :

- When $\operatorname{Re} z_{3} \in(\pi, 2 \pi)$, the SL fibration $F^{t}$ for $t \in(0,1]$ locally resembles the SL fibration $F$ of Theorem 5.1 near $\left(0,0, z_{3}\right) \mathbb{Z}$, with singularities of multiplicity 1 and increasing type.

- When $\operatorname{Re} z_{3} \in(0, \pi)$, the SL fibration $F^{t}$ for $t \in(0,1]$ locally resembles the SL fibration $F^{\prime}$ of Theorem 5.3 near $\left(0,0, z_{3}\right) \mathbb{Z}$, with singularities of multiplicity 1 and decreasing type.

- When $\operatorname{Re} z_{3}=0$, the SL fibration $F^{t}$ for $t \in(0,1]$ locally resembles the SL fibration $\hat{F}$ of Theorem 6.5 near $\left(0,0, z_{3}\right) \mathbb{Z}$, with singularities of multiplicity 2 and maximum type.

- When $\operatorname{Re} z_{3}=\pi$, the SL fibration $F^{t}$ for $t \in(0,1]$ locally resembles an analogue of the SL fibration $\hat{F}$ of Theorem 6.5 near $\left(0,0, z_{3}\right) \mathbb{Z}$, with singularities of multiplicity 2 and minimum type.

Thus, we have not found any new kinds of local singular behaviour of SL fibrations in this section, we have just assembled those already discussed in $\S 5$ and $\S 6$ in a model with more interesting global topology.

\section{Global behaviour of SL fibrations.}

We are now ready to state our picture (still conjectural and incomplete) of what SL fibrations of generic almost Calabi-Yau 3-folds look like, if indeed they exist. Rather than starting from scratch, we begin in $\S 8.1$ by reviewing the elegant picture of smooth SL fibrations $f: M \rightarrow B$, which has been built up largely by Mark Gross and Wei-Dong Ruan. Then in $\S 8.2$ we explain how to modify the Gross-Ruan picture under a small generic deformation of $M$. Finally, in $\S 8.3$ we draw some conclusions about the SYZ Conjecture. 


\subsection{The Gross-Ruan picture of smooth SL fibrations.}

Here is a review of the expected properties of smooth SL fibrations of CalabiYau 3-folds. Our principal sources are Gross [4, §3] and Ruan [21, §7] for the topology of the singular fibres, and Gross [5, §1] and Ruan [22, §9] for the monodromy matrices. A concise statement may be found in the 'Precise SYZ mirror conjecture' of Ruan [22, §9].

Let $f: M \rightarrow \mathcal{S}^{3}$ be a smooth SL fibration, with fibres $N_{b}=f^{-1}(b)$, and generic fibre $T^{3}$. For generic such fibrations, the discriminant $\Delta$ is thought to be a trivalent graph, made up of smooth edges, and vertices of two kinds, which we shall refer to as positive and negative. The topology and local monodromy for each kind of singular fibre are as follows.

(a) Edges. Let $\gamma$ be an edge in $\Delta$, and $b \in \gamma$. Then $N_{b}$ has the topology of $T^{3}$ with $T^{2}$ collapsed to an $\mathcal{S}^{1}$, and may be written $\Sigma \times \mathcal{S}^{1}$, where $\Sigma$ is a $T^{2}$ with an $\mathcal{S}^{1}$ collapsed to a point, or equivalently an $\mathcal{S}^{2}$ with two points identified. These fibres are called type $(2,2)$ by Gross and type $I$ by Ruan. They have Euler characteristic zero.

The monodromy about each edge $\gamma$ in $\Delta$, acting on $H_{1}\left(T^{3} ; \mathbb{Z}\right) \cong \mathbb{Z}^{3}$, is

$$
\left(\begin{array}{lll}
1 & 1 & 0 \\
0 & 1 & 0 \\
0 & 0 & 1
\end{array}\right)
$$

with respect to a suitable basis of $H_{1}\left(T^{3} ; \mathbb{Z}\right)$.

(b) Positive vertices. Let $b$ be a positive vertex in $\Delta$. Then $N_{b}$ has the topology of $T^{3}$ with $T^{2}$ collapsed to a point. It has Euler characteristic 1 . These fibres are called type $(1,2)$ by Gross and type $I I I$ by Ruan.

The monodromies around the three edges $\gamma_{1}, \gamma_{2}, \gamma_{3}$ meeting at $b$ are

$$
\left(\begin{array}{lll}
1 & 0 & 0 \\
1 & 1 & 0 \\
0 & 0 & 1
\end{array}\right), \quad\left(\begin{array}{ccc}
1 & 0 & 0 \\
0 & 1 & 0 \\
-1 & 0 & 1
\end{array}\right) \text { and }\left(\begin{array}{ccc}
1 & 0 & 0 \\
-1 & 1 & 0 \\
1 & 0 & 1
\end{array}\right)
$$

with respect to a suitable basis of $H_{1}\left(T^{3} ; \mathbb{Z}\right)$.

The smooth SL fibration of Example 4.4 is a local model for the fibration $f$ near the singular point of a positive singular fibre.

(c) Negative vertices. Let $b$ be a negative vertex in $\Delta$. Then Ruan $[21$, $\S 7]$ gives two different possible topologies for $N_{b}$, which he calls type $I I$ and 
type $\tilde{I I}$. His type $\tilde{I I}$ topology agrees with Gross' proposed type $(2,1)$ fibre $[4$, $\S 3]$.

Both fibres are constructed by taking a fibration $\pi: T^{3} \rightarrow T^{2}$ with fibre $\mathcal{S}^{1}$, and collapsing the fibres to points over a graph $\Gamma$ in $T^{2}$. In the type $I I$ case $\Gamma$ has three edges and two vertices, and in the type $\tilde{I I}$ case it has two edges and one vertex. In both cases $N_{b}$ has Euler characteristic -1 .

The monodromies around the three edges $\gamma_{1}, \gamma_{2}, \gamma_{3}$ meeting at $b$ are

$$
\left(\begin{array}{lll}
1 & 1 & 0 \\
0 & 1 & 0 \\
0 & 0 & 1
\end{array}\right),\left(\begin{array}{ccc}
1 & 0 & -1 \\
0 & 1 & 0 \\
0 & 0 & 1
\end{array}\right) \text { and }\left(\begin{array}{ccc}
1 & -1 & 1 \\
0 & 1 & 0 \\
0 & 0 & 1
\end{array}\right)
$$

with respect to a suitable basis of $H_{1}\left(T^{3} ; \mathbb{Z}\right)$.

At present, to the author's knowledge, there is no known local model for a smooth special Lagrangian fibration (or even a smooth Lagrangian fibration) in the neighbourhood of a codimension three singular point of a negative singular fibre. The author conjectures that no such local model exists. If this is the case then smooth SL fibrations may not exist on general Calabi-Yau 3-folds, even with a very nongeneric choice of almost Calabi-Yau metric.

We will refer to the singular fibres over positive and negative vertices as positive and negative singular fibres respectively. Our notation of positive and negative vertices was suggested by David Morrison, and refers to the sign of the Euler characteristic of the singular fibres. Gross' notation refers to the Betti numbers $\left(b^{1}, b^{2}\right)$ of the singular fibres.

\subsection{Modification of this picture for generic ACY 3-folds.}

Now we shall something about what special Lagrangian fibrations of generic almost Calabi-Yau 3-folds might look like. Suppose we start with a smooth Gross-Ruan fibration $f: M \rightarrow B$, either of a nongeneric almost Calabi-Yau 3 -fold or of the degenerate large complex structure limit, and make a small perturbation to a generic almost Calabi-Yau 3-fold. What happens to the fibration?

Near a nonsingular fibre $N_{b}=f^{-1}(b)$ of $f$, the fibration should remain nonsingular, and the local geometry unchanged. The interesting question is what happens to the singular fibres of $f$. The following is the author's best guess, on the assumption that SL fibrations are well-behaved in the generic case. We preface it with some remarks on monodromy and coordinates on the moduli space. 
Let $f: M \rightarrow B$ be an SL fibration. By Theorem 2.6, near a nonsingular fibre $N_{b} \cong T^{3}$ the moduli space of deformations of $N_{b}$ is isomorphic to $H^{1}\left(N_{b} ; \mathbb{R}\right) \cong \mathbb{R}^{3}$. But this moduli space is $B$, and so near any point in $B \backslash \Delta_{f}$ we have natural affine coordinates modelled on $H^{1}\left(T^{3} ; \mathbb{R}\right)$.

However, near a singular fibre $N_{b}$ the situation is more complicated because of the monodromy action. Let $N_{b^{\prime}}$ be a nonsingular fibre near $N_{b}$. Let $\Gamma_{b}$ be the set of monodromies of loops in $B \backslash \Delta_{f}$ based at $b^{\prime}$ and staying in a small neighbourhood of $b$. Then $\Gamma_{b}$ is a group acting on $H_{1}\left(N_{b^{\prime}} ; \mathbb{Z}\right)$ and $H^{1}\left(N_{b^{\prime}} ; \mathbb{R}\right)$. Roughly speaking, near $b$ we can regard $B$ as a kind of quotient of $H^{1}\left(N_{b^{\prime}} ; \mathbb{R}\right)$ by $\Gamma_{b}$, so that $B$ is a kind of orbifold, with the topology of a 3 -manifold, but not the smooth structure.

In what follows, as long as we make use of only $\Gamma_{b^{-}}$-invariant objects, we can think of $B$ as being locally like $\mathbb{R}^{3}$ and mostly ignore the monodromy action. We shall represent elements of $H_{1}\left(N_{b^{\prime}} ; \mathbb{Z}\right)$ by column vectors, and elements of $H^{1}\left(N_{b^{\prime}} ; \mathbb{R}\right)$ by row vectors, upon which the monodromy matrices of equations (46)-(48) act by left and right multiplication respectively.

Here is a conjectural picture of how generic SL fibrations work around perturbations of the Gross-Ruan singular fibres described in (a)-(c) of $\S 8.1$.

(a) Edges. The author conjectures that under small deformations, the 'edges' $\gamma$ in the Gross-Ruan picture will thicken out into thin 'ribbons' $R$ of the kind described in $\S 7$. They are closed subsets of hyperplanes in $B$ defined locally by $[\omega] \cdot[D]=0$, where $[\omega]$ is the relative de Rham cohomology class in $H^{1}\left(M, N_{b} ; \mathbb{R}\right)$ and $[D]$ a relative homology class in $H_{1}\left(M, N_{b} ; \mathbb{Z}\right)$ depending on the edge, which will be represented by an even number of holomorphic discs $D$ for generic $b \in B$, as we discussed in $\S 5.1$ and $\S 6.1$.

Choose an identification $H_{1}\left(N_{b} ; \mathbb{Z}\right) \cong \mathbb{Z}^{3}$ such that the monodromy around $\gamma$ is as in (46). Then calculation using the local model of $\S 7$ shows that $[\partial D] \in H_{1}\left(N_{b} ; \mathbb{Z}\right)$ should be identified with $\pm\left(\begin{array}{lll}1 & 0 & 0\end{array}\right)^{T}$ in $\mathbb{Z}^{3}$. Also, $B$ is locally identified with $H^{1}\left(N_{b} ; \mathbb{R}\right) \cong \mathbb{R}^{3}$ up to monodromy, and $R$ lies in the monodromy-invariant hyperplane $\left\{\left(0, x_{2}, x_{3}\right): x_{j} \in \mathbb{R}\right\}$.

The situation described in $\S 7$, in which the generic singular fibre has two singular points, is only the simplest possibility. In general we expect the generic singular fibre to contain an even number of singular points, divided equally into two kinds. In codimension one on the ribbon these singular points can appear or disappear in pairs of different kinds, and the edge of the ribbon is where the last two singular points disappear. 
(b) Positive vertices. For positive vertices in the Gross-Ruan picture, the monodromy matrices of (47) all fix the vectors

$$
\mathbf{v}_{1}=\left(\begin{array}{l}
0 \\
1 \\
0
\end{array}\right), \quad \mathbf{v}_{2}=\left(\begin{array}{c}
0 \\
0 \\
-1
\end{array}\right) \quad \text { and } \quad \mathbf{v}_{3}=\left(\begin{array}{c}
0 \\
-1 \\
1
\end{array}\right)
$$

in $H_{1}\left(N_{b^{\prime}} ; \mathbb{Z}\right)$ and the direction $(100)$ in $H^{1}\left(N_{b^{\prime}} ; \mathbb{R}\right)$.

In a generic perturbation of a Gross-Ruan fibration near a positive vertex, the three edges in $\Delta_{f}$ should thicken out into 'ribbons' $R_{1}, R_{2}, R_{3}$ lying in the three hyperplanes

$$
\begin{gathered}
H_{1}=\left\{\left(x_{1}, 0, x_{3}\right): x_{j} \in \mathbb{R}\right\}, \quad H_{2}=\left\{\left(x_{1}, x_{2}, 0\right): x_{j} \in \mathbb{R}\right\} \\
\text { and } \quad H_{3}=\left\{\left(x_{1}, x_{2}, x_{3}\right): x_{j} \in \mathbb{R}, \quad x_{2}=x_{3}\right\},
\end{gathered}
$$

which are the hyperplanes dual to $\mathbf{v}_{1}, \mathbf{v}_{2}, \mathbf{v}_{3}$, and intersect in $\left\{\left(x_{1}, 0,0\right)\right.$ : $\left.x_{1} \in \mathbb{R}\right\}$. The ribbons $R_{1}, R_{2}, R_{3}$ intersect in a bounded subinterval of this line, as sketched in Figure 1.

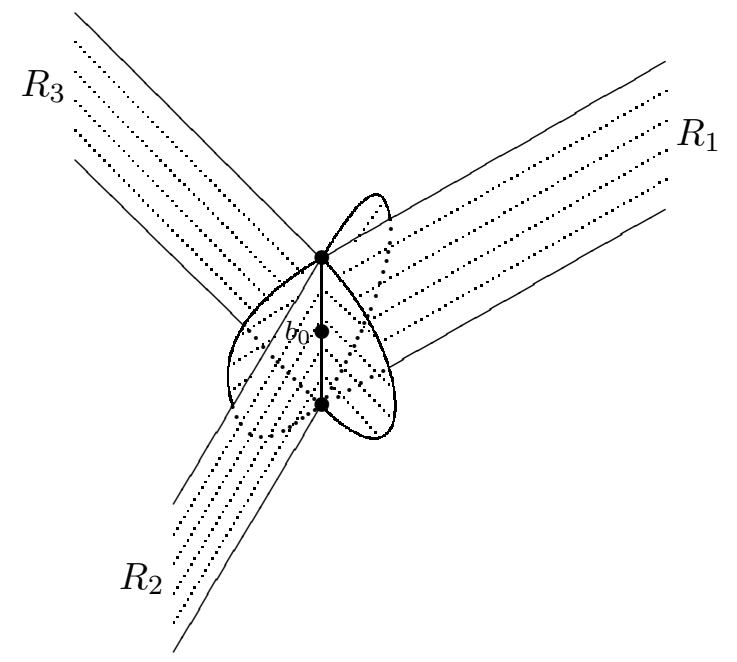

Figure 1: Discriminant locus near a perturbation of a positive vertex

There are two obvious ways for this to happen, in which either $R_{1} \cap R_{2} \cap$ $R_{3}$ is part of the boundary of each $R_{j}$, or the ribbons $R_{j}$ extend a little way beyond their intersection $R_{1} \cap R_{2} \cap R_{3}$. The author thinks that the latter option is what actually happens, as in Figure 1.

For generic points in the intersection $R_{1} \cap R_{2} \cap R_{3}$ the singularities of the fibres are just finitely many points modelled locally on the $T^{2}$-cone $C$ 
of (29). These are divided into three kinds, corresponding to the ribbons $R_{1}, R_{2}, R_{3}$, according to the homology class of the $\mathcal{S}^{1}$ in $T^{3}$ that collapses to a point.

However, at certain special points $b_{0}$ in $R_{1} \cap R_{2} \cap R_{3}$ there will be a new kind of codimension three singularity, when two or three of these singular points of different kinds come together. The author does not have a local model for this singularity, but topologically it may involve a cone on a genus 2 surface. There must be at least one such singular fibre, as it is necessary for the monodromy to work out.

When $N_{b^{\prime}}$ is a generic nonsingular fibre near the ribbon $R_{j}$, there should exist an even number of holomorphic discs $D_{j}$ in $M$ whose boundary $\partial D_{j}$ in $N_{b^{\prime}}$ has homology class $\pm \mathbf{v}_{j}$ in $H_{1}\left(N_{b^{\prime}} ; \mathbb{Z}\right) \cong \mathbb{Z}^{3}$. Singularities develop when the area of $D_{j}$ shrinks to zero, which happens on the hyperplane $H_{j}$ in $B$.

(c) Negative vertices. For negative vertices, the monodromy matrices of (48) all fix the vector $(100)^{T}$ in $H_{1}\left(N_{b^{\prime}} ; \mathbb{Z}\right)$ and the hyperplane $\left\{\left(0, x_{2}, x_{3}\right)\right.$ : $\left.x_{j} \in \mathbb{R}\right\}$ in $H^{1}\left(N_{b^{\prime}} ; \mathbb{R}\right)$. In a generic perturbation of a Gross-Ruan fibration near a negative vertex, the three edges in $\Delta_{f}$ should thicken out into 'ribbons' which all lie in the same hyperplane $H$ in $B$, isomorphic to $\left\{\left(0, x_{2}, x_{3}\right): x_{j} \in\right.$ $\mathbb{R}\}$ in $H^{1}\left(N_{b^{\prime}} ; \mathbb{R}\right)$. The three ribbons merge together to make a letter $Y$ shape in $H$, as sketched in Figure 2 .

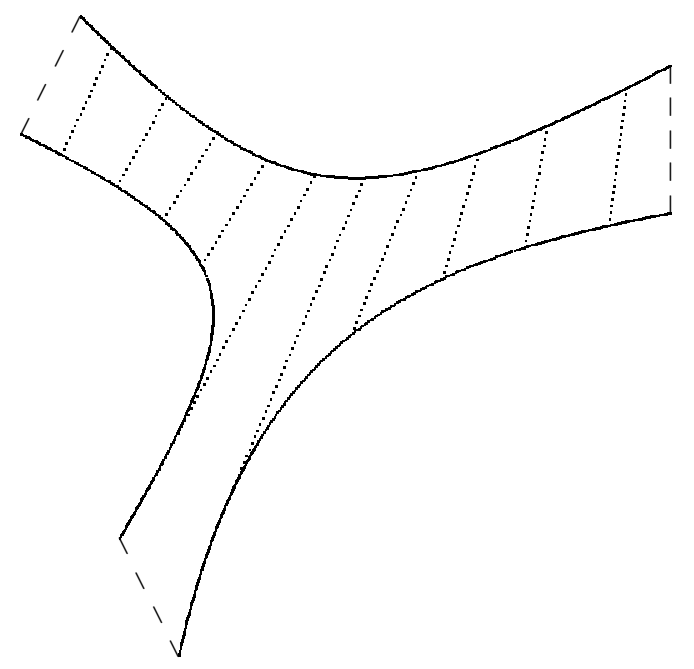

Figure 2: Discriminant locus near a perturbation of a negative vertex

When $N_{b^{\prime}}$ is a generic nonsingular fibre near this part of $\Delta_{f}$, there should exist an even number of homologous holomorphic discs $D$ in $M$ whose bound- 
aries $\partial D$ in $N_{b^{\prime}}$ have homology class $\pm\left(\begin{array}{lll}1 & 0 & 0\end{array}\right)^{T}$ in $H_{1}\left(N_{b^{\prime}} ; \mathbb{Z}\right) \cong \mathbb{Z}^{3}$. Singularities develop when the area of $D$ shrinks to zero, which happens on the hyperplane $H$ in $B$.

Calculations by the author, along the lines of $\S 7$ but more complicated, show that one can put together a fibration with the topological properties we want using only the local models of $\S 5$ and $\S 6$. There is no need to include any other kind of singular point.

The author is fairly confident about parts (a) and (c), but less happy about part (b). In fact, Ruan's Lagrangian fibrations by gradient flow look quite like parts (a) and (c) in the relevant regions. Another option in part (b) is that there could be a new kind of codimension two singularity along the line segment $R_{1} \cap R_{2} \cap R_{3}$.

\subsection{Conclusions.}

If the speculations of $\S 8.2$ are correct, they have important consequences for the SYZ Conjecture. Positive and negative singular fibres are expected to be dual to one another under the mirror transform. That is, if we have dual smooth SL fibrations $f: M \rightarrow B$ and $\hat{f}: \hat{M} \rightarrow B$ as in the SYZ conjecture, then positive vertices in the discriminant $\Delta_{f}$ of $f$ in $B$ should coincide with negative vertices in the discriminant $\Delta_{\hat{f}}$ of $\hat{f}$, and vice versa. This follows as the monodromy matrices in (47) are the transposes of those in (48).

However, after a small generic perturbation of $f$ and $\hat{f}$ near such a vertex in $B$, it is clear from Figures 1 and 2 that the discriminant loci $\Delta_{f}$ and $\Delta_{\hat{f}}$ can no longer be identified, because they are not homeomorphic. On this basis we make the following conjecture.

Conjecture 8.1. Let $M, \hat{M}$ be generic mirror Calabi-Yau 3-folds. Then even if there do exist special Lagrangian fibrations $f: M \rightarrow B$ and $\hat{f}: \hat{M} \rightarrow$ $\hat{B}$, it is not in general possible to homeomorphically identify the bases $B$ and $\hat{B}$ of the fibrations in a way that identifies the discriminants $\Delta_{f}, \Delta_{\hat{f}}$ of $f, \hat{f}$, and so that the nonsingular fibres of $f, \hat{f}$ are 3 -tori with dual homology.

This contradicts the version of the SYZ Conjecture stated in the introduction, and some of the stronger forms of the SYZ Conjecture that people have written down so far. If it is true then it will limit the scope of any eventual final formulation of the SYZ Conjecture.

My feeling is that while the SYZ Conjecture is clearly morally true, it is probably not literally true of genuine special Lagrangian fibrations of 
holonomy SU(3) Calabi-Yau 3-folds, except in some limiting sense in the large complex structure limit.

Furthermore, I believe that the Gross-Ruan picture of smooth SL fibrations is probably asymptotically true of general SL fibrations in the large complex structure limit, so that in a family of Calabi-Yau 3-folds with SL fibrations approaching the large complex structure limit, the 2-dimensional discriminants will collapse down onto 1-dimensional trivalent graphs.

Therefore, a better way to formulate the SYZ Conjecture might be in terms of SL fibrations of 1-parameter families of mirror Calabi-Yau 3-folds $M_{t}, \hat{M}_{t}$ for $t \in(0, \epsilon)$, which both approach the large complex structure limit as $t \rightarrow 0$. A similar conclusion is reached by Gross in [6, $\S 4]$, based partly on the first version of this paper.

\section{References.}

[1] J.J. Duistermaat, On global action-angle coordinates, Comm. Pure Appl. Math. 33 (1980), 687-706.

[2] D. Gilbarg and N.S. Trudinger, Elliptic Partial Differential Equations of Second Order, Classics in Mathematics, Springer-Verlag, Berlin, 2001.

[3] M. Gross, Special Lagrangian fibrations I: Topology. In M.-H. Saito, Y. Shimizu, and K. Ueno, editors, Integrable Systems and Algebraic Geometry, pages 156-193, World Scientific, Singapore, 1998. alggeom/9710006.

[4] M. Gross, Special Lagrangian fibrations II: Geometry. In Differential Geometry inspired by String Theory, Surveys in Differential Geometry 5, pages 341-403, International Press, 1999. math.AG/9809072.

[5] M. Gross, Topological mirror symmetry, Invent. math. 144 (2001), 75137. math.AG/9909015.

[6] M. Gross, Examples of special Lagrangian fibrations. In K. Fukaya, Y.G. Oh, K. Ono and G. Tian, editors, Symplectic geometry and mirror symmetry (Seoul, 2000), pages 81-109, World Scientific, Singapore, 2001. math.AG/0012002.

[7] R. Harvey and H.B. Lawson, Calibrated geometries, Acta Mathematica 148 (1982), 47-157.

[8] D.D. Joyce, Lectures on Calabi-Yau and special Lagrangian geometry, math.DG/0108088, 2001. Published, with extra material, as Part I of M. 
Gross, D. Huybrechts and D. Joyce, Calabi-Yau Manifolds and Related Geometries, Universitext series, Springer, Berlin, 2003.

[9] D.D. Joyce, U(1)-invariant special Lagrangian 3-folds. I. Nonsingular solutions, math.DG/0111324, 2001. To appear in Advances in Mathematics.

[10] D.D. Joyce, U(1)-invariant special Lagrangian 3-folds. II. Existence of singular solutions, math.DG/0111326, 2001.

[11] D.D. Joyce, U(1)-invariant special Lagrangian 3-folds. III. Properties of singular solutions, math.DG/0204343, 2002.

[12] D.D. Joyce, U(1)-invariant special Lagrangian 3-folds and special Lagrangian fibrations, Turkish J. Math. 27 (2003), 99-114. math.DG/ 0206016.

[13] D.D. Joyce, Special Lagrangian submanifolds with isolated conical singularities. I. Regularity, math.DG/0211294, version 3, 2003.

[14] D.D. Joyce, Special Lagrangian submanifolds with isolated conical singularities. II. Moduli spaces, math.DG/0211295, version 3, 2003.

[15] D.D. Joyce, Special Lagrangian submanifolds with isolated conical singularities. III. Desingularization, the unobstructed case, math.DG/0302355, version 2, 2003.

[16] D.D. Joyce, Special Lagrangian submanifolds with isolated conical singularities. IV. Desingularization, obstructions and families, math.DG/0302356, version 2, 2003.

[17] D.D. Joyce, Special Lagrangian submanifolds with isolated conical singularities. V. Survey and applications, math.DG/0303272, 2003. To appear in the Journal of Differential Geometry.

[18] R.C. McLean, Deformations of calibrated submanifolds, Communications in Analysis and Geometry 6 (1998), 705-747.

[19] F. Morgan, Geometric Measure Theory, A Beginner's Guide, Academic Press, San Diego, 1995.

[20] W.-D. Ruan, Lagrangian tori fibration of toric Calabi-Yau manifold I, math.DG/9904012, 1999. 
[21] W.-D. Ruan, Lagrangian tori fibration of toric Calabi-Yau manifold III: symplectic topological SYZ mirror construction for general quintics, math.DG/9909126, 1999.

[22] W.-D. Ruan, Lagrangian torus fibration and mirror symmetry of Calabi-Yau hypersurface in toric variety, math.DG/0007028, 2000.

[23] A. Strominger, S.-T. Yau, and E. Zaslow, Mirror symmetry is T-duality, Nuclear Physics B479 (1996), 243-259. hep-th/9606040.

LinCOLN COLlege

OXFORD, OX1 3DR

dominic.joyce@lincoln.ox.ac.uk

Received February 9, 2001. 